\title{
FINITE ELEMENT METHODS IN LOCAL ACTIVE CONTROL OF SOUND
}

\author{
ALFREDO BERMÚDEZ*, PABLO GAMALLO*, AND RODOLFO RODRÍGUEZ ${ }^{\dagger}$
}

\begin{abstract}
The active control of sound is analyzed in the framework of the mathematical theory of optimal control. After setting the problem in the frequency domain, we deal with the state equation, which is a Helmholtz partial differential equation. We show existence of a unique solution and analyze a finite element approximation when the source term is a Dirac delta measure. Two optimization problems are successively considered. The first one concerns the choice of phases and amplitudes of the actuators to minimize the noise at the sensors location. The second one consists in determining the optimal actuators placement. Both problems are then numerically solved. Error estimates are settled and numerical results for some tests are reported.
\end{abstract}

Key words. Dissipative acoustics, noise reduction, active control, optimal control problem, finite element approximation.

AMS subject classifications. 49J20, 49K20, 65N15

1. Introduction. Noise reduction is an important problem in acoustical and environmental engineering. While passive methods are good for middle and high frequencies, they are not efficient for low ones. However, the latter can be significantly reduced by active control techniques. This is an old concept that has generated increasing interest during the last years due to the development of fast digital signal processors (DSP). It is based on the principle of destructive interference of waves: an opposite pressure is generated by a secondary source to cancel an undesired noise. In order to achieve a significant reduction, this source must produce, with great precision, an equal amplitude but inverted replica of the noise to be canceled. Applications of these techniques can be used, for instance, to reduce noise in aircrafts or cars.

Reference books on this subject are [3] and [11]. The general principles of active control of noise was described in an early patent by Leug in 1936. A microphone detects the undesired noise and provides an input signal to an electronic control system. The transfer from the microphone to the loudspeaker is adjusted so that the sound wave generated will destructively interfere the noise to be canceled.

In this paper we state the problem of active control of noise in the framework of the optimal control theory of distributed systems and present its mathematical and numerical analysis. For the sake of simplicity we consider that the noise to be canceled has one single frequency, although it is also possible to control broad-band or even non-periodic noises. Two problems are successively considered. In a first step complex amplitudes are taken as control variables with the objective of minimizing the pressure at some particular points in the domain. In a second step the loud-speakers location is optimized with respect to the same objective function. A third step that is not included here would consist in determining the microphones location in view of minimizing the global noise, i.e., the norm of the pressure in the whole domain under consideration rather than at some finite number of points.

The outline of the paper is as follows. In Section 2 we introduce the physical problem and pose it in the framework of the optimal control theory. In Section 3

\footnotetext{
*Departamento de Matemática Aplicada, Universidade de Santiago de Compostela, 15782 Santiago de Compostela, Spain. Partially funded by MCYT, Spain, under grant DPI2001-1613-C02-02 and Xunta de Galicia research project PGIDT02PXIC20701PN.

${ }^{\dagger} \mathrm{GI}^{2} \mathrm{MA}$, Departamento de Ingeniería Matemática, Universidad de Concepción, Casilla 160-C, Concepción, Chile. Partially funded by FONDAP in Applied Mathematics, Chile.
} 
we analyze the state equation. Although our main concern is when the inner source terms are Dirac delta measures, to tackle this problem we analyze first the same equation with data in $L^{2}$. We prove existence and uniqueness of solution and analyze its regularity, including some local $W^{2, \infty}$ a priori estimates which are used in the following section. In Section 4 we introduce a finite element method to approximate the state equation. Once more we study first the case with $L^{2}$ data and then with Dirac delta measures. In both cases we prove $L^{2}$ and pointwise error estimates. In Section 5 we state an optimal control problem to determine the optimal amplitudes of the actuators and show that it is well posed. Then we approximate it by using the finite element approximation of the state equation introduced in the previous section. Next, we prove an error estimate for the approximate optimal control. In Section 6 we report some numerical results which confirm our theoretical assertions. In Section 7 we study how to determine the optimal location of the actuators, again in the framework of the optimal control theory. We prove existence of an optimal control in this case and settle the optimality conditions. Finally, we report the results of some numerical experiments.

2. Mathematical model. The optimal control problem. Let $\Omega \subset \mathbb{R}^{n}$ ( $n=2$ or 3 ) be a bounded, convex, $2 \mathrm{D}$ polygonal or $3 \mathrm{D}$ polyhedral domain enclosing a non-dissipative acoustic fluid (i.e., inviscid, compressible, and barotropic). The propagation of acoustic waves in this domain is modeled by the well known equation

$$
\frac{1}{c^{2}} \frac{\partial^{2} P(x, t)}{\partial t^{2}}-\Delta P(x, t)=F(x, t) \quad \text { in } \Omega,
$$

where $P$ is the pressure fluctuation, $c$ the sound speed, and $F$ an inner source term. In our case, $F$ will correspond to the secondary source of noise produced by loudspeakers, which will be the control variable. Moreover, there is a primary noise source acting on a part $\Gamma_{\mathrm{N}}$ of the boundary of the domain, $\partial \Omega$, which is modeled by

$$
\frac{\partial P(x, t)}{\partial \boldsymbol{n}}=G(x, t) \quad \text { on } \Gamma_{\mathrm{N}},
$$

where $\boldsymbol{n}$ is an outward unit normal vector to $\partial \Omega$. This means that normal displacements are imposed on $\Gamma_{\mathrm{N}}=\bigcup_{j=1}^{J} \Gamma_{\mathrm{N}}^{j}$, where $\Gamma_{\mathrm{N}}^{1}, \ldots, \Gamma_{\mathrm{N}}^{J}$ denote the plane faces of $\Gamma_{\mathrm{N}}$. In practice, it corresponds to the effect of an external vibration source transmitted to the enclosure $\Omega$ by the vibrations of some of the walls. Finally, we assume that the rest of the boundary $\Gamma_{\mathrm{z}}:=\partial \Omega \backslash \bar{\Gamma}_{\mathrm{N}}=\bigcup_{k=1}^{K} \Gamma_{\mathrm{z}}^{k}$ is formed by damping plane walls $\Gamma_{\mathrm{z}}^{1}, \ldots, \Gamma_{\mathrm{z}}^{K}$ characterized by a frequency-dependent wall impedance $Z(\omega)$. We assume that $\left|\Gamma_{\mathrm{Z}}\right|^{\mathrm{z}}>0$ and $\left|\Gamma_{\mathrm{N}}\right|>0$, too.

In this paper we consider that $G$ is a harmonic source with angular frequency $\omega \in \mathbb{R}, \omega \neq 0$. Hence, the secondary source $F$ must be chosen also harmonic with the same frequency, i.e.,

$$
G(x, t)=\operatorname{Re}\left[g(x) \mathrm{e}^{-i \omega t}\right], \quad F(x, t)=\operatorname{Re}\left[f(x) \mathrm{e}^{-i \omega t}\right],
$$

where $g(x)$ and $f(x)$ are complex functions which correspond to the respective complex amplitudes. Actually, their modulus are the physical amplitudes while their arguments are the phase angles. Since the model is linear, the stationary solution of the wave equation is also harmonic with the same frequency:

$$
P(x, t)=\operatorname{Re}\left[p(x) \mathrm{e}^{-i \omega t}\right] .
$$


In such case, we are led to the following Helmholtz problem, whose solution is the complex pressure amplitude:

$$
\left\{\begin{aligned}
-\Delta p-\left(\frac{\omega}{c}\right)^{2} p & =f & & \text { in } \Omega \\
\frac{\partial p}{\partial \boldsymbol{n}} & =\frac{i \omega \rho}{Z(\omega)} p & & \text { on } \Gamma_{\mathrm{Z}} \\
\frac{\partial p}{\partial \boldsymbol{n}} & =g & & \text { on } \Gamma_{\mathrm{N}}
\end{aligned}\right.
$$

where $\rho$ is the density of the fluid and $Z(\omega) \in \mathbb{C}$ is the wall impedance which is given by

$$
Z(\omega):=\beta(\omega)+\frac{\alpha(\omega)}{\omega} i .
$$

The boundary condition on $\Gamma_{z}$ allows modeling the behavior of absorbing viscoelastic materials covering the enclosure walls which are typically used as passive systems to reduce low-frequency noise. The frequency-dependent coefficients $\alpha(\omega)$ and $\beta(\omega)$ are related to the viscous and elastic responses of the isolating material, respectively. Both are strictly positive functions of the angular frequency $\omega$. However, in what follows, we will only assume that $\beta(\omega) \neq 0$.

In our case, the secondary source $f$ will be a linear combination of $N$ Dirac delta measures supported at some given points, $y_{1}, \ldots, y_{N} \in \Omega$ with complex amplitudes $u_{1}, \ldots, u_{N}$ to be determined:

$$
f=\sum_{i=1}^{N} u_{i} \delta_{y_{i}}, \quad \text { with } u_{i} \in \mathbb{C}, i=1, \ldots, N .
$$

This amounts to considering loud-speakers as acoustic monopoles (see for instance [11]).

In order to state the noise active control as an optimal control problem, we make the following choices:

- the state of the system is given by the pressure $p(x)$ in the domain $\Omega$;

- the control variable $\boldsymbol{u}$ is the vector of complex amplitudes of the loud-speakers (actuators),

$$
\boldsymbol{u}:=\left(u_{1}, \ldots, u_{N}\right) \in \mathbb{C}^{N},
$$

which define the source term $f$ in (2.1) by means of $(2.2)$;

- the set of admissible controls is a convex closed set $U_{\text {ad }} \subseteq \mathbb{C}^{N}$;

- the model of the system relating the control variable to the state is the Helmholtz problem (2.1);

- the observation $\boldsymbol{z}$ is the set of pressure values at $M$ microphones (sensors) located at given points $w_{1}, \ldots, w_{M} \in \Omega$,

$$
\boldsymbol{z}(\boldsymbol{u}):=\left(p\left(w_{1}\right), \ldots, p\left(w_{M}\right)\right) \in \mathbb{C}^{M},
$$

where, for $\boldsymbol{u} \in \mathbb{C}^{N}, p$ denotes the solution of problem (2.1) with $f$ given by (2.2); in the next section it will be shown that evaluating pressure at points $w_{i} \in \Omega$ makes sense as long as they do not coincide with the locations of the actuators; 
- the cost function to be minimized depends on the observation and eventually on the cost of the control itself, namely,

$$
J(\boldsymbol{u}):=\frac{1}{2}\|\boldsymbol{z}(\boldsymbol{u})\|^{2}+\frac{\nu}{2}\|\boldsymbol{u}\|^{2}
$$

where $\nu \geq 0$ is a weighting factor, and $\|\cdot\|$ denotes the Euclidean norm in $\mathbb{C}^{N}$ or $\mathbb{C}^{M}$.

Thus we are led to the following optimal control problem:

Find $\boldsymbol{u}^{\mathrm{op}} \in U_{\mathrm{ad}}$ such that

$$
J\left(\boldsymbol{u}^{\mathrm{op}}\right)=\inf _{\boldsymbol{u} \in U_{\mathrm{ad}}} J(\boldsymbol{u})
$$

Any solution $\boldsymbol{u}^{\text {op }}$ of this minimization problem will be called an optimal control.

3. State equation. In this section we prove existence and uniqueness of solution of the state equation and analyze its regularity, which will be used to study the optimal control problem. Our goal is the Helmholtz equation with singular data because in our case $f$ is a linear combination of Dirac delta measures. However, we tackle this problem by first analyzing the same equation with data in $L^{2}(\Omega)$.

3.1. Data in $L^{2}(\Omega)$. We consider the Helmholtz problem (2.1) with $f \in L^{2}(\Omega)$ and $g \in L^{2}\left(\Gamma_{\mathrm{N}}\right)$. Multiplying the first equation by a test function $q \in H^{1}(\Omega)$, taking into account the boundary conditions, and using a Green's formula, we obtain the following weak formulation of (2.1):

Find $p \in H^{1}(\Omega)$ such that

$$
\begin{array}{rl}
\int_{\Omega} \nabla p \cdot \nabla \bar{q} d x-\frac{i \omega \rho}{Z(\omega)} \int_{\Gamma_{\mathrm{Z}}} & p \bar{q} d \Gamma-\left(\frac{\omega}{c}\right)^{2} \int_{\Omega} p \bar{q} d x \\
& =\int_{\Omega} f \bar{q} d x+\int_{\Gamma_{\mathrm{N}}} g \bar{q} d \Gamma \quad \forall q \in H^{1}(\Omega) .
\end{array}
$$

We denote by $a_{\omega}$ the sesquilinear continuous form in $H^{1}(\Omega)$ appearing in the left hand side of this problem:

$$
a_{\omega}(p, q):=\int_{\Omega} \nabla p \cdot \nabla \bar{q} d x-\frac{i \omega \rho}{Z(\omega)} \int_{\Gamma_{\mathrm{Z}}} p \bar{q} d \Gamma-\left(\frac{\omega}{c}\right)^{2} \int_{\Omega} p \bar{q} d x, \quad p, q \in H^{1}(\Omega) .
$$

It is clear that $a_{\omega}$ is not positive definite and therefore the Lax-Milgram Lemma can not be applied to show existence and uniqueness of solution. Instead, we show below that $a_{\omega}$ satisfies a Gårding's inequality. Then, according to Fredholm's alternative, uniqueness implies existence of solution.

To prove uniqueness we consider the homogeneous problem

$$
\widetilde{p} \in H^{1}(\Omega): \quad a_{\omega}(\widetilde{p}, q)=0 \quad \forall q \in H^{1}(\Omega) .
$$

Due to the damping viscous term $\beta \neq 0$ of the wall impedance $Z$, from the viewpoint of physics, no solution $\widetilde{p} \neq 0$ of problem (3.2) should be expected when $\omega \in \mathbb{R}, \omega \neq 0$. Indeed, we have the following result.

Lemma 3.1. If $\left|\Gamma_{\mathrm{Z}}\right|>0, \omega \in \mathbb{R}, \omega \neq 0$, and $\beta \neq 0$, then $\widetilde{p}=0$ is the unique solution of problem (3.2). 
Proof. Let $\widetilde{p}$ be a solution of (3.2). By choosing $q=\widetilde{p}$ in (3.2) we obtain

$$
A-\frac{i \omega \rho}{\beta+\frac{\alpha}{\omega} i} B-\left(\frac{\omega}{c}\right)^{2} C=A-\frac{\alpha \rho}{\beta^{2}+\frac{\alpha^{2}}{\omega^{2}}} B-\left(\frac{\omega}{c}\right)^{2} C-i \frac{\omega \rho \beta}{\beta^{2}+\frac{\alpha^{2}}{\omega^{2}}} B=0,
$$

where $A:=\int_{\Omega}|\nabla \widetilde{p}|^{2} d x, B:=\int_{\Gamma_{\mathrm{Z}}}|\widetilde{p}|^{2} d \Gamma$, and $C=\int_{\Omega}|\widetilde{p}|^{2} d x$ are real numbers. Then, the imaginary part must vanish too. Hence, for $\beta \neq 0$ and $\omega \neq 0$, we have $B=0$ and, consequently, $\widetilde{p}=0$ on $\Gamma_{\mathrm{z}}$. Then, by taking test functions $q \in \mathcal{C}^{\infty}(\bar{\Omega})$ in (3.2), we obtain that $\widetilde{p}$ satisfies

$$
\left\{\begin{aligned}
-\Delta \widetilde{p} & =\left(\frac{\omega}{c}\right)^{2} \widetilde{p} & & \text { in } \Omega, \\
\frac{\partial \widetilde{p}}{\partial \boldsymbol{n}} & =0 & & \text { on } \partial \Omega, \\
\widetilde{p} & =0 & & \text { on } \Gamma_{\mathrm{z}} .
\end{aligned}\right.
$$

According to these equations, if $\widetilde{p} \neq 0$, then it would be an eigenfunction of the Laplace operator satisfying simultaneously Neumann and Dirichlet homogeneous conditions on $\Gamma_{\mathrm{z}}$, which is not possible because of the Unique Prolongation Theorem. Thus $\widetilde{p}=0$ and we conclude the proof. $\square$

On the other hand, the following lemma shows that the sesquilinear form $a_{\omega}$ satisfies a Gårding's inequality.

Lemma 3.2. There exist strictly positive constants $\gamma$ and $C_{\omega}$, the latter depending on $\omega \in \mathbb{R}$, such that

$$
\left|a_{\omega}(q, q)+C_{\omega}\|q\|_{L^{2}(\Omega)}^{2}\right| \geq \gamma\|q\|_{H^{1}(\Omega)}^{2} \quad \forall q \in H^{1}(\Omega) .
$$

Proof. For all $q \in H^{1}(\Omega)$ and $\omega \in \mathbb{R}$, we have

$$
\operatorname{Re}\left[a_{\omega}(q, q)\right]=\|q\|_{H^{1}(\Omega)}^{2}-\frac{\alpha \rho}{\beta^{2}+\frac{\alpha^{2}}{\omega^{2}}}\|q\|_{L^{2}\left(\Gamma_{\mathrm{Z}}\right)}^{2}-\left(1+\frac{\omega^{2}}{c^{2}}\right)\|q\|_{L^{2}(\Omega)}^{2} .
$$

Thus, if $\alpha \leq 0$, then (3.3) holds with $\gamma=1$ and $C_{\omega}=1+\omega^{2} / c^{2}$. Otherwise, from the trace theorem (see for instance [2]), $\exists C>0$ such that

$$
\|q\|_{L^{2}\left(\Gamma_{\mathrm{Z}}\right)} \leq C\|q\|_{L^{2}(\Omega)}^{1 / 2}\|q\|_{H^{1}(\Omega)}^{1 / 2} \quad \forall q \in H^{1}(\Omega) .
$$

Hence, $\forall \varepsilon>0$ we have that

$$
\|q\|_{L^{2}\left(\Gamma_{\mathrm{Z}}\right)}^{2} \leq \varepsilon\|q\|_{H^{1}(\Omega)}^{2}+\frac{C^{2}}{4 \varepsilon}\|q\|_{L^{2}(\Omega)}^{2} \quad \forall q \in H^{1}(\Omega) .
$$

Then, the choice $\varepsilon=\left(\beta^{2}+\alpha^{2} / \omega^{2}\right) /(2 \alpha \rho)>0$ leads to

$$
\operatorname{Re}\left[a_{\omega}(q, q)+C_{\omega}\|q\|_{L^{2}(\Omega)}^{2}\right] \geq \gamma\|q\|_{H^{1}(\Omega)}^{2}
$$

with $\gamma:=1 / 2$ and $C_{\omega}:=1+\omega^{2} / c^{2}+C^{2} /\left(8 \varepsilon^{2}\right)$. Therefore, since $|\cdot| \geq \operatorname{Re}(\cdot)$, we end the proof.

Now we are able to conclude the following existence, uniqueness, and regularity of solution result for the Helmholtz problem given above. From now on $C$ denotes a strictly positive constant not necessarily the same at each occurrence. 
Theorem 3.3. Let $\omega \in \mathbb{R}, \omega \neq 0, \beta \neq 0, f \in L^{2}(\Omega)$ and $g \in L^{2}\left(\Gamma_{\mathrm{N}}\right)$. Then, problem (3.1) has a unique solution $p \in H^{1}(\Omega)$. Moreover, if $\left.g\right|_{\Gamma_{\mathrm{N}}^{j}} \in H^{1 / 2}\left(\Gamma_{\mathrm{N}}^{j}\right)$, $j=1, \ldots, J$, then $p \in H^{2}(\Omega)$ and the following estimate holds:

$$
\|p\|_{H^{2}(\Omega)} \leq C\left[\|f\|_{L^{2}(\Omega)}+\sum_{j=1}^{J}\|g\|_{H^{1 / 2}\left(\Gamma_{\mathrm{N}}^{j}\right)}\right] .
$$

Proof. Uniqueness has been proved in Lemma 3.1. Because of Lemma 3.2 we know that the problem satisfies Fredholm's alternative (see for instance Theorem 6.5.15 of [10]). Then, existence is a consequence of uniqueness. Moreover, because of the Open Mapping Theorem,

$$
\|p\|_{H^{1}(\Omega)} \leq C\left[\|f\|_{L^{2}(\Omega)}+\|g\|_{L^{2}\left(\Gamma_{\mathrm{N}}\right)}\right] .
$$

Next, by testing (3.1) with functions $q \in \mathcal{C}^{\infty}(\bar{\Omega})$ we obtain that $p$ satisfies

$$
\left\{\begin{aligned}
-\Delta p+p & =\left(1+\frac{\omega^{2}}{c^{2}}\right) p+f & & \text { in } \Omega \\
\frac{\partial p}{\partial \boldsymbol{n}} & =\frac{i \omega \rho}{Z(\omega)} p & & \text { on } \Gamma_{\mathrm{z}} \\
\frac{\partial p}{\partial \boldsymbol{n}} & =g & & \text { on } \Gamma_{\mathrm{N}} .
\end{aligned}\right.
$$

Since the domain $\Omega$ is a convex $2 \mathrm{D}$ polygon or $3 \mathrm{D}$ polyhedron, the standard a priori estimate for this Neumann problem (see [5] and [9]) yields

$$
\|p\|_{H^{2}(\Omega)} \leq C\left[\|p\|_{L^{2}(\Omega)}+\|f\|_{L^{2}(\Omega)}+\sum_{k=1}^{K}\|p\|_{H^{1 / 2}\left(\Gamma_{\mathrm{Z}}^{k}\right)}+\sum_{j=1}^{J}\|g\|_{H^{1 / 2}\left(\Gamma_{\mathrm{N}}^{j}\right)}\right],
$$

where $C$ is a constant independent of $f, g$, and $p$. Then, (3.4) is a direct consequence of this inequality and (3.5).

REMARK 1. The convexity assumption on $\Omega$ is used only to obtain the estimate (3.4) and the analogous one for the Green's function (3.11) below. Similar results are valid for smooth non-convex domains, too.

3.2. Dirac delta measures. Let us now consider the Helmholtz problem (2.1) with homogeneous Neumann boundary data and inner source $f=\delta_{y}$ being the Dirac delta measure supported at an inner point $y \in \Omega$. Its solution is the Green's function $G^{y} \in L^{2}(\Omega)$ of problem (2.1):

$$
\left\{\begin{aligned}
-\Delta G^{y}-\left(\frac{\omega}{c}\right)^{2} G^{y} & =\delta_{y} & & \text { in } \Omega \\
\frac{\partial G^{y}}{\partial \boldsymbol{n}} & =\frac{i \omega \rho}{Z(\omega)} G^{y} & & \text { on } \Gamma_{\mathrm{z}} \\
\frac{\partial G^{y}}{\partial \boldsymbol{n}} & =0 & & \text { on } \Gamma_{\mathrm{N}}
\end{aligned}\right.
$$

where the first equation must be understood in the sense of distributions. 
It is simple to show that this problem has a unique solution. Indeed, let $\Phi^{y}$ be the fundamental solution of the Helmholtz equation in the whole space; namely, the solution of

$$
-\Delta \Phi^{y}-\left(\frac{\omega}{c}\right)^{2} \Phi^{y}=\delta_{y} \quad \text { in } \mathbb{R}^{n}
$$

This fundamental solution is explicitly known (see for instance [6]):

$$
\Phi^{y}(x):= \begin{cases}\frac{1}{4} Y_{0}\left(\frac{\omega}{c}|x-y|\right), & \text { if } n=2, \\ \frac{\cos \left(\frac{\omega}{c}|x-y|\right)}{4 \pi|x-y|}, & \text { if } n=3,\end{cases}
$$

where, $Y_{0}$ denotes the zero-order second-kind Bessel function. It clearly satisfies $\Phi^{y} \in \mathcal{C}^{\infty}(\mathbb{R} \backslash\{y\})$ and $\left.\Phi^{y}\right|_{\Omega} \in L^{2}(\Omega)$. Then $G^{y}$ is a solution of (3.6) if and only if $G^{y}=\left.\Phi^{y}\right|_{\Omega}+p^{y}$, with $p^{y}$ satisfying

$$
\left\{\begin{aligned}
-\Delta p^{y}-\left(\frac{\omega}{c}\right)^{2} p^{y} & =0 & & \text { in } \Omega \\
\frac{\partial p^{y}}{\partial \boldsymbol{n}} & =\frac{i \omega \rho}{Z(\omega)} p^{y}+\frac{i \omega \rho}{Z(\omega)} \Phi^{y}-\frac{\partial \Phi^{y}}{\partial \boldsymbol{n}} & & \text { on } \Gamma_{\mathrm{Z}} \\
\frac{\partial p^{y}}{\partial \boldsymbol{n}} & =-\frac{\partial \Phi^{y}}{\partial \boldsymbol{n}} & & \text { on } \Gamma_{\mathrm{N}}
\end{aligned}\right.
$$

The variational formulation of this problem consists in finding $p^{y} \in H^{1}(\Omega)$ such that

$$
a_{\omega}\left(p^{y}, q\right)=\int_{\Gamma_{\mathrm{Z}}}\left[\frac{i \omega \rho}{Z(\omega)} \Phi^{y}-\frac{\partial \Phi^{y}}{\partial \boldsymbol{n}}\right] \bar{q} d \Gamma-\int_{\Gamma_{\mathrm{N}}} \frac{\partial \Phi^{y}}{\partial \boldsymbol{n}} \bar{q} d \Gamma \quad \forall q \in H^{1}(\Omega),
$$

with $a_{\omega}$ as defined above. Then, the arguments in the proof of Theorem 3.3 allow us to conclude that this problem has a unique solution and, hence, problem (3.6) too. Moreover, these arguments also show that $p^{y} \in H^{2}(\Omega)$ and, furthermore,

$$
\left\|p^{y}\right\|_{H^{2}(\Omega)} \leq C\left[\sum_{k=1}^{K}\left\|\frac{i \omega \rho}{Z(\omega)} \Phi^{y}-\frac{\partial \Phi^{y}}{\partial \boldsymbol{n}}\right\|_{H^{1 / 2}\left(\Gamma_{\mathrm{Z}}^{k}\right)}+\sum_{j=1}^{J}\left\|\frac{\partial \Phi^{y}}{\partial \boldsymbol{n}}\right\|_{H^{1 / 2}\left(\Gamma_{\mathrm{N}}^{j}\right)}\right] .
$$

Consequently $p^{y}$ is a continuous function and, hence, $G^{y}=\Phi^{y}+p^{y}$ is continuous in $\Omega \backslash\{y\}$. This shows that evaluating the pressure at a point $w \in \Omega$ where a microphone is located makes sense as long as $w \neq y$. Therefore, the control problem (2.4) is wellposed whenever the sets of sensors and actuators locations do not intersect.

Furthermore, if $d>0$ is such that $\mathcal{B}_{d}(y):=\left\{x \in \mathbb{R}^{n}:|x-y|<d\right\} \subset \subset \Omega$, then $\left\|\Phi^{y}\right\|_{H^{2}\left(\Omega \backslash \overline{\mathcal{B}}_{d}(y)\right)}$ is bounded by a constant which depends on $d$. Thus, from the estimate (3.10) we have that

$$
\left\|G^{y}\right\|_{H^{2}\left(\Omega \backslash \overline{\mathcal{B}}_{d}(y)\right)} \leq\left\|\Phi^{y}\right\|_{H^{2}\left(\Omega \backslash \overline{\mathcal{B}}_{d}(y)\right)}+\left\|p^{y}\right\|_{H^{2}(\Omega)} \leq C,
$$

with $C$ depending on $d$, too.

To end this subsection we present an alternative characterization of the solution of problem (3.6) obtained by transposition techniques. This will be used to prove convergence in $L^{2}(\Omega)$ of the numerical scheme introduced in the following section. 
To this goal note that given $q \in L^{2}(\Omega)$, the adjoint problem to (3.6) reads

$$
\left\{\begin{aligned}
-\Delta r-\left(\frac{\omega}{c}\right)^{2} r & =q & & \text { in } \Omega \\
\frac{\partial r}{\partial \boldsymbol{n}} & =-\frac{i \omega \rho}{\bar{Z}(\omega)} r & & \text { on } \Gamma_{\mathrm{Z}} \\
\frac{\partial r}{\partial \boldsymbol{n}} & =0 & & \text { on } \Gamma_{\mathrm{N}} .
\end{aligned}\right.
$$

In spite of the conjugate in the coefficient of the middle equation, Theorem 3.3 applies to this problem since it has been proved with the only assumptions that $\omega \neq 0$ and $\beta \neq 0$. Hence (3.12) has a unique solution which satisfies $r \in H^{2}(\Omega)$ and

$$
\|r\|_{H^{2}(\Omega)} \leq C\|q\|_{L^{2}(\Omega)} .
$$

In what follows we prove that the solution $G^{y}$ of (3.6) is the unique function in $L^{2}(\Omega)$ which satisfies

$$
\int_{\Omega} G^{y} \bar{q} d x=\left\langle\delta_{y}, r\right\rangle \quad \forall q \in L^{2}(\Omega)
$$

Indeed, standard computations (see for instance Chapter 2.2.4 of [7]) shows that

$$
\left\langle\delta_{y}, r\right\rangle=\int_{\Omega} \Phi^{y}\left(-\Delta \bar{r}-\frac{\omega^{2}}{c^{2}} \bar{r}\right) d x+\int_{\partial \Omega}\left(\Phi^{y} \frac{\partial \bar{r}}{\partial \boldsymbol{n}}-\frac{\partial \Phi^{y}}{\partial \boldsymbol{n}} \bar{r}\right) d \Gamma .
$$

On the other hand, integration by parts in (3.9) with $r$ as a test function yields

$$
\int_{\Omega} p^{y}\left(-\Delta \bar{r}-\frac{\omega^{2}}{c^{2}} \bar{r}\right) d x-\frac{i \omega \rho}{Z(\omega)} \int_{\Gamma_{\mathrm{Z}}}\left(p^{y}+\Phi^{y}\right) \bar{r} d \Gamma+\int_{\partial \Omega}\left(p^{y} \frac{\partial \bar{r}}{\partial \boldsymbol{n}}+\frac{\partial \Phi^{y}}{\partial \boldsymbol{n}} \bar{r}\right) d \Gamma=0 .
$$

Then, by adding these two equations we obtain

$$
\left\langle\delta_{y}, r\right\rangle=\int_{\Omega} G^{y}\left(-\Delta \bar{r}-\frac{\omega^{2}}{c^{2}} \bar{r}\right) d x-\frac{i \omega \rho}{Z(\omega)} \int_{\Gamma_{\mathrm{z}}} G^{y} \bar{r} d \Gamma+\int_{\partial \Omega} G^{y} \frac{\partial \bar{r}}{\partial \boldsymbol{n}}=\int_{\Omega} G^{y} \bar{q} d x,
$$

where we have used the three equations of (3.12) for the last equality. Thus we conclude (3.14).

3.3. Local $W^{2, \infty}$ a priori estimates. The following lemma yields $L^{\infty}$ local a priori estimates for the second derivatives of the solutions of problems (3.1) and (3.6). These bounds will be used in the following section to obtain pointwise error estimates for the finite element method proposed therein to solve these problems.

Lemma 3.4. Let $D_{0}$ and $D_{1}$ be disjoint open subsets of $\Omega$ satisfying $D_{i} \subset \subset \Omega$, $i=0,1$. Let $d>0$ be such that $\operatorname{dist}\left(D_{i}, \partial \Omega\right) \geq d, i=0,1$, and $\operatorname{dist}\left(D_{0}, D_{1}\right) \geq d$. Then:

1. For each $y \in D_{0}$, the solution of problem (3.6) satisfies $\left.G^{y}\right|_{D_{1}} \in W^{2, \infty}\left(D_{1}\right)$ and there exists a constant $C>0$ depending on $d$ such that

$$
\left\|G^{y}\right\|_{W^{2, \infty}\left(D_{1}\right)} \leq C \quad \forall y \in D_{0}
$$


2. Let $f \in L^{2}(\Omega)$ be such that $\operatorname{supp}(f) \subset D_{0}$. Let $g \in L^{2}\left(\Gamma_{\mathrm{N}}\right)$ be such that $\left.g\right|_{\Gamma_{\mathrm{N}}^{j}} \in H^{1 / 2}\left(\Gamma_{\mathrm{N}}^{j}\right), j=1, \ldots, J$. Let $p$ be the solution of problem (3.1). Then, there exists a constant $C>0$ depending on $d$ such that

$$
\|p\|_{W^{2, \infty}\left(D_{1}\right)} \leq C\left[\|f\|_{L^{2}(\Omega)}+\sum_{j=1}^{J}\|g\|_{H^{1 / 2}\left(\Gamma_{\mathrm{N}}^{j}\right)}\right] .
$$

Proof. Consider the following subsets of $\Omega: D_{2}:=\left\{x \in \mathbb{R}^{n}: \operatorname{dist}\left(x, D_{1}\right)<\frac{d}{4}\right\}$ and $D_{3}:=\left\{x \in \mathbb{R}^{n}: \operatorname{dist}\left(x, D_{1}\right)<\frac{d}{2}\right\}$. Then, $\operatorname{dist}\left(D_{3}, \partial \Omega\right) \geq \frac{d}{2}$ and $\operatorname{dist}\left(D_{0}, D_{3}\right) \geq$ $\frac{d}{2}$. Let $\chi$ be a $\mathcal{C}^{\infty}$ cut-off real function supported in $D_{3}$ such that $\left.\chi\right|_{D_{2}}=1$ and $\|\chi\|_{W^{2, \infty}\left(\mathbb{R}^{n}\right)}$ is bounded by a constant depending on $d$.

Given $y \in D_{0}$, we write the solution of problem (3.6) in the form $G^{y}=\left.\Phi^{y}\right|_{\Omega}+p^{y}$, with $\Phi^{y}$ given by (3.7) and $p^{y}$ being the solution of (3.8). Explicit differentiation of (3.7) shows that, for all $y \in D_{0}$, $\operatorname{since} \operatorname{dist}\left(y, D_{3}\right) \geq \frac{d}{2},\left\|\Phi^{y}\right\|_{W^{2, \infty}\left(D_{3}\right)}$ is bounded by a constant depending on $d$ but not on $y$. So, to prove the first part of the theorem we only need to estimate $\left\|p^{y}\right\|_{W^{2, \infty}\left(D_{1}\right)}$.

Given $z \in D_{1}$, let $G^{z}$ be the solution of problem (3.6) with $y$ substituted by $z$. Then, we have

$$
p^{y}(z)=\left\langle\delta_{z}, \chi p^{y}\right\rangle=\left\langle-\Delta G^{z}-\frac{\omega^{2}}{c^{2}} G^{z}, \chi p^{y}\right\rangle=\left\langle G^{z},-\Delta\left(\chi p^{y}\right)-\frac{\omega^{2}}{c^{2}} \chi p^{y}\right\rangle,
$$

and

$$
\begin{aligned}
-\Delta\left(\chi p^{y}\right)-\frac{\omega^{2}}{c^{2}} \chi p^{y} & =\chi\left(-\Delta p^{y}-\frac{\omega^{2}}{c^{2}} p^{y}\right)-2 \nabla \chi \cdot \nabla p^{y}-(\Delta \chi) p^{y} \\
& =-\left[2 \nabla \chi \cdot \nabla p^{y}+(\Delta \chi) p^{y}\right] .
\end{aligned}
$$

Hence, $\operatorname{supp}\left(-\Delta\left(\chi p^{y}\right)-\frac{\omega^{2}}{c^{2}} \chi p^{y}\right) \subset \operatorname{supp}(\nabla \chi) \subset D_{3} \backslash \bar{D}_{2}$, and thus

$$
p^{y}(z)=-\int_{D_{3} \backslash \bar{D}_{2}} G^{z}(x)\left[2 \nabla \chi(x) \cdot \nabla \bar{p}^{y}(x)+\Delta \chi(x) \bar{p}^{y}(x)\right] d x .
$$

Because of the symmetry of the involved operator, the Green's function is symmetric, i.e., $G^{z}(x)=G^{x}(z) \quad \forall x, z \in \Omega: x \neq z$. Then, by differentiating the expression above we obtain $\forall \alpha \in \mathbb{N}^{n}$,

$$
D_{z}^{\alpha} p^{y}(z)=-\int_{D_{3} \backslash \bar{D}_{2}} D_{x}^{\alpha} G^{z}(x)\left[2 \nabla \chi(x) \cdot \nabla \bar{p}^{y}(x)+\Delta \chi(x) \bar{p}^{y}(x)\right] d x .
$$

Consequently, by (3.11) and (3.10), we have $\forall \alpha \in \mathbb{N}^{n}$ such that $|\alpha|:=\sum_{l=1}^{n} \alpha_{l} \leq 2$,

$$
\begin{aligned}
\left|D_{z}^{\alpha} p^{y}(z)\right| & \leq\left\|G^{z}\right\|_{H^{2}\left(D_{3} \backslash \bar{D}_{2}\right)}\|\chi\|_{W^{2, \infty}\left(D_{3} \backslash \bar{D}_{2}\right)}\left\|p^{y}\right\|_{H^{1}\left(D_{3} \backslash \bar{D}_{2}\right)} \\
& \leq C\left[\sum_{k=1}^{K}\left\|\frac{i \omega \rho}{Z(\omega)} \Phi^{y}-\frac{\partial \Phi^{y}}{\partial \boldsymbol{n}}\right\|_{H^{1 / 2}\left(\Gamma_{\mathrm{Z}}^{k}\right)}+\sum_{j=1}^{J}\left\|\frac{\partial \Phi^{y}}{\partial \boldsymbol{n}}\right\|_{H^{1 / 2}\left(\Gamma_{\mathrm{N}}^{j}\right)}\right] \leq C,
\end{aligned}
$$

with $C$ depending on $d$, but not on the particular point $z \in D_{1}$. Thus we conclude the proof of the first part of the lemma.

For the second one, let $f \in L^{2}(\Omega)$ with $\operatorname{supp}(f) \subset D_{0}$. We proceed exactly as above and use that

$$
-\Delta(\chi p)-\frac{\omega^{2}}{c^{2}}(\chi p)=\chi\left(-\Delta p-\frac{\omega^{2}}{c^{2}} p\right)-2 \nabla \chi \cdot \nabla p-\Delta \chi p=-(2 \nabla \chi \cdot \nabla p+\Delta \chi p),
$$


because of $-\Delta p-\frac{\omega^{2}}{c^{2}} p=f$ and $\operatorname{supp}(f) \cap \operatorname{supp} \chi=\emptyset$. Then we obtain from (3.11) and Theorem 3.3,

$$
\begin{aligned}
\left|D_{z}^{\alpha} p(z)\right| & \leq\left|\int_{D_{3} \backslash \bar{D}_{2}} D_{x}^{\alpha} G^{z}(x)\left[2 \nabla \chi(x) \cdot \nabla \bar{p}^{y}(x)+\Delta \chi(x) \bar{p}^{y}(x)\right] d x\right| \\
& \leq\left\|G^{z}\right\|_{H^{2}\left(D_{3} \backslash \bar{D}_{2}\right)}\|\chi\|_{W^{2, \infty}\left(D_{3} \backslash \bar{D}_{2}\right)}\|p\|_{H^{1}\left(D_{3} \backslash \bar{D}_{2}\right)} \\
& \leq C\left[\|f\|_{L^{2}(\Omega)}+\sum_{j=1}^{J}\|g\|_{H^{1 / 2}\left(\Gamma_{\mathrm{N}}^{j}\right)}\right] \quad \forall \alpha \in \mathbb{N}^{n}:|\alpha| \leq 2,
\end{aligned}
$$

with $C$ again depending on $d$ but not on the particular point $z \in D_{1}$. Thus we conclude the proof.

4. Numerical approximation of the state equation. For the construction of the finite element spaces we consider a quasi-uniform family of shape-regular triangulations $\left\{\mathcal{T}_{h}\right\}_{h>0}$ of $\Omega$. More precisely, for each element $T \in \mathcal{T}_{h}$ ( $T$ a $2 \mathrm{D}$ triangle or a 3D tetrahedron) we associate two parameters: $h_{T}$ and $\rho_{T}$. The first one denotes the diameter of $T$ and the second one the diameter of the largest ball contained in $T$. We denote $h:=\max _{T \in \mathcal{T}_{h}} h_{T}$ and make the following hypothesis of regularity of the triangulation: there exist positive constants $\sigma_{1}$ and $\sigma_{2}$ such that

$$
\frac{h_{T}}{\rho_{T}} \leq \sigma_{1}, \quad \frac{h}{h_{T}} \leq \sigma_{2}, \quad \forall T \in \mathcal{T}_{h}, \forall h>0 .
$$

We associate with each triangulation $\mathcal{T}_{h}$ a finite element space $\mathcal{V}_{h}$ which consists of functions globally continuous in $\Omega$ and linear on each element $T \in \mathcal{T}_{h}$. Then, the discrete problem associated with problem (3.1) is the following:

Find $p_{h} \in \mathcal{V}_{h}$ such that

$$
a_{\omega}\left(p_{h}, q_{h}\right)=\left\langle f, q_{h}\right\rangle+\int_{\Gamma_{\mathrm{N}}} g \bar{q}_{h} d \Gamma \quad \forall q_{h} \in \mathcal{V}_{h} .
$$

Notice that this problem is well defined for $f \in L^{2}(\Omega)$ as well as for $f$ given by (2.2), because the functions in $\mathcal{V}_{h}$ are continuous.

4.1. Data in $L^{2}(\Omega)$. Again, to tackle the numerical approximation when the source term is a Dirac delta measure, we consider first the problem with data in $L^{2}(\Omega)$.

Since $a_{\omega}$ is continuous and satisfies the Gårding's inequality (3.3), and the continuous problem (3.1) has a unique solution, the following existence and approximation result is readily obtained from [12].

THEOREM 4.1. Given $f \in L^{2}(\Omega)$ and $g \in L^{2}\left(\Gamma_{\mathrm{N}}\right)$, let $p$ be the solution of problem (3.1). Then, there exists $h_{0}>0$ such that, for all $h \in\left(0, h_{0}\right]$, problem (4.1) has a unique solution $p_{h}$. Moreover, if $\left.g\right|_{\Gamma_{\mathrm{N}}^{j}} \in H^{1 / 2}\left(\Gamma_{\mathrm{N}}^{j}\right), j=1, \ldots, J$, then the following estimate holds:

$$
\left\|p-p_{h}\right\|_{L^{2}(\Omega)} \leq C h^{2}\left[\|f\|_{L^{2}(\Omega)}+\sum_{j=1}^{J}\|g\|_{H^{1 / 2}\left(\Gamma_{\mathrm{N}}^{j}\right)}\right] .
$$

Since the observation $\boldsymbol{z}(\boldsymbol{u})$ consists of point values of the solution of problem (3.1), a pointwise error estimate will be used in the following section to obtain an 
error bound for the approximate control. To this aim, we have the following result which is a consequence of the interior maximum norm estimates proved in [13].

TheOREM 4.2. Given $f \in L^{2}(\Omega)$ such that $\operatorname{supp}(f) \subset \subset \Omega$ and $g \in L^{2}\left(\Gamma_{\mathrm{N}}\right)$ such that $\left.g\right|_{\Gamma_{\mathrm{N}}^{j}} \in H^{1 / 2}\left(\Gamma_{\mathrm{N}}^{j}\right), j=1, \ldots, J$, let $p$ be the solution of problem (3.1). Given $w \in \Omega \backslash \operatorname{supp}(f)$, let $d>0$ be such that $\operatorname{dist}(w, \partial \Omega) \geq d$, $\operatorname{dist}(w, \operatorname{supp}(f)) \geq d$, and $\operatorname{dist}(\operatorname{supp}(f), \partial \Omega) \geq d$. Let $h_{0}>0$ be such that, for all $h \in\left(0, h_{0}\right]$, problem (4.1) has a unique solution $p_{h}$. Then, there exist strictly positive constants $h_{1}<h_{0}$ and $C$, both depending on $d$, such that the following pointwise error estimate holds $\forall h \in\left(0, h_{1}\right]$ :

$$
\left|p(w)-p_{h}(w)\right| \leq C h^{2} \ln \left(\frac{1}{h}\right)\left[\|f\|_{L^{2}(\Omega)}+\sum_{j=1}^{J}\|g\|_{H^{1 / 2}\left(\Gamma_{\mathrm{N}}^{j}\right)}\right] .
$$

Proof. Let $D:=\mathcal{B}_{d / 8}(w), D_{1}:=\mathcal{B}_{d / 4}(w)$, and $D_{2}:=\mathcal{B}_{d / 2}(w)$. Then $D_{2} \subset \subset \Omega$ and $D_{2} \cap \operatorname{supp}(f)=\emptyset$, with $\operatorname{dist}\left(D_{2}, \partial \Omega\right) \geq \frac{d}{2}$ and $\operatorname{dist}\left(D_{2}, \operatorname{supp}(f)\right) \geq \frac{d}{2}$.

Now, for $h<h_{0}$, since $p$ and $p_{h}$ are solutions of (3.1) and (4.1), respectively, $\forall q_{h} \in \mathcal{V}_{h}$ with $\operatorname{supp}\left(q_{h}\right) \subset D_{1}$ there holds

$$
\int_{\Omega} \nabla\left(p-p_{h}\right) \cdot \nabla \bar{q}_{h} d x-\left(\frac{\omega}{c}\right)^{2} \int_{\Omega}\left(p-p_{h}\right) \bar{q}_{h} d x=0 .
$$

Then, according to Theorem 5.1 in [13], there exist $C>0$ and $h_{1}>0$ such that $\forall h \in\left(0, h_{1}\right]$ and $\forall q_{h} \in \mathcal{V}_{h}$ the following inequality holds:

$$
\left\|p-p_{h}\right\|_{L^{\infty}(D)} \leq C \ln \left(\frac{1}{h}\right)\left[\left\|p-q_{h}\right\|_{L^{\infty}\left(D_{1}\right)}+\left\|p-p_{h}\right\|_{L^{2}\left(D_{1}\right)}\right] .
$$

Because of Lemma 3.4 (Part 2) and the standard error estimate for the Lagrange interpolation (see for instance [2]), if $h<d / 4$, then

$$
\inf _{q_{h} \in \mathcal{V}_{h}}\left\|p-q_{h}\right\|_{L^{\infty}\left(D_{1}\right)} \leq C h^{2}\|p\|_{W^{2, \infty}\left(D_{2}\right)} \leq C\left[\|f\|_{L^{2}(\Omega)}+\sum_{j=1}^{J}\|g\|_{H^{1 / 2}\left(\Gamma_{\mathrm{N}}^{j}\right)}\right] .
$$

Thus, the theorem follows from the last two inequalities and Theorem 4.1. $\square$

4.2. Dirac delta measures. We consider now problem (4.1) with $g=0$ and $f$ being a Dirac delta measure:

$$
G_{h}^{y} \in \mathcal{V}_{h}: \quad a_{\omega}\left(G_{h}^{y}, q_{h}\right)=\left\langle\delta_{y}, q_{h}\right\rangle \quad \forall q_{h} \in \mathcal{V}_{h} .
$$

This is a discretization of problem (3.6). Let us recall that it is well defined because the functions in $\mathcal{V}_{h}$ are continuous. Then, by proceeding as in the previous subsection, it can be shown that $\exists h_{0}$ such that $\forall h \in\left(0, h_{0}\right]$, this problem has a unique solution. Here and thereafter, $h_{0}$ denotes a maximum mesh-size not necessarily the same at each occurrence.

To show convergence in $L^{2}(\Omega)$, we are going to use the scheme proposed for elliptic problems in [14] (see also [4]). This scheme allows splitting the approximation error into two parts: the first one due to the error in the approximation of the Dirac delta measure by $L^{2}(\Omega)$ functions and the second one due to the approximation error of the Helmholtz equation with data in $L^{2}(\Omega)$. 
Consider the following auxiliary variational problem:

$$
\widetilde{G} \in H^{1}(\Omega): \quad a_{\omega}(\widetilde{G}, q)=\left\langle\delta_{h}, q\right\rangle \quad \forall q \in H^{1}(\Omega),
$$

where $\delta_{h} \in L^{2}(\Omega)$ is an approximation of $\delta_{y}$ satisfying the following properties:

1. $\left\langle\delta_{h}, q_{h}\right\rangle=\int_{\Omega} \delta_{h} \bar{q}_{h} d x=\bar{q}_{h}(y) \quad \forall q_{h} \in \mathcal{V}_{h}$,

2. $\left\|\delta_{h}\right\|_{L^{2}(\Omega)} \leq C h^{-n / 2}$,

3. $\left\|\delta_{y}-\delta_{h}\right\|_{H^{-2}(\Omega)} \leq C h^{2-n / 2}$,

4. $\delta_{h}=0$ outside the elements in which $y$ lies.

A construction of such $\delta_{h}$ is given in [14].

We use the triangular inequality to separate the error in two terms:

$$
\left\|G^{y}-G_{h}^{y}\right\|_{L^{2}(\Omega)} \leq\left\|G^{y}-\widetilde{G}\right\|_{L^{2}(\Omega)}+\left\|\widetilde{G}-G_{h}^{y}\right\|_{L^{2}(\Omega)} .
$$

From property 1 above we conclude that $\delta_{y}$ and $\delta_{h}$ are identical functionals on $\mathcal{V}_{h}$. Consequently $G_{h}^{y}$ can be seen as a finite element approximation of $\widetilde{G}$, too. Therefore, from Theorem 4.1 and property 2 of $\delta_{h}$, we obtain the following approximation result for $h$ sufficiently small:

$$
\left\|\widetilde{G}-G_{h}^{y}\right\|_{L^{2}(\Omega)} \leq C h^{2}\left\|\delta_{h}\right\|_{L^{2}(\Omega)} \leq C h^{2-n / 2} .
$$

To estimate the remaining term, note that by property 4 above $\delta_{h}$ has its support included in a certain set $D \subset \subset \Omega$, for $h$ sufficiently small. Then, we can apply to $\widetilde{G}$ the arguments of the transposition technique that allows us to prove (3.14). By so doing, we obtain that $\forall q \in L^{2}(\Omega)$, if $r \in H^{2}(\Omega)$ is the solution of (3.12), then

$$
\int_{\Omega}\left(G^{y}-\widetilde{G}\right) \bar{q}=\left\langle\delta_{y}-\delta_{h}, r\right\rangle .
$$

Hence, by taking $q=G^{y}-\widetilde{G}$ and using the a priori estimate (3.13), we have

$$
\left\|G^{y}-\widetilde{G}\right\|_{L^{2}(\Omega)}^{2} \leq\left\|\delta_{y}-\delta_{h}\right\|_{H^{-2}(\Omega)}\|r\|_{H^{2}(\Omega)} \leq C\left\|\delta_{y}-\delta_{h}\right\|_{H^{-2}(\Omega)}\left\|G^{y}-\widetilde{G}\right\|_{L^{2}(\Omega)} .
$$

Consequently, from property 3 of $\delta_{h}$ we have,

$$
\left\|G^{y}-\widetilde{G}\right\|_{L^{2}(\Omega)} \leq C\left\|\delta_{y}-\delta_{h}\right\|_{H^{-2}(\Omega)} \leq C h^{2-n / 2} .
$$

Thus we have proved the following result.

THEOREM 4.3. For $y \in \Omega$ let $G^{y} \in L^{2}(\Omega)$ be the solution of problem (3.6). There exists $h_{0}>0$ such that, for all $h \in\left(0, h_{0}\right]$, problem (4.2) has a unique solution $G_{h}^{y}$. Moreover, there exists a strictly positive constant $C$ such that the following error bound holds:

$$
\left\|G^{y}-G_{h}^{y}\right\|_{L^{2}(\Omega)} \leq C h^{2-n / 2}
$$

Next, we prove an error estimate for $\left|G^{y}(w)-G_{h}^{y}(w)\right|$, for $w \neq y$. This will be used in the following section to obtain an error bound for the approximate control. A similar result with the explicit dependence of the constant of the estimate on $|w-y|$ has been proved in Theorem 6.1(i) of [13], but for a coercive second-order linear 
operator with homogeneous Neumann boundary conditions on a smooth domain. The additional hypotheses of this theorem were used only to obtain an explicit estimate for the corresponding Green's function (see (4.9) of this reference). However, to the best of the authors' knowledge, such estimate is not available for our Helmholtz problem with mixed Neumann-Robin boundary conditions on a convex polyhedron. Instead, we will use the local maximum norm a priori estimate proved in Lemma 3.4 (Part 1) to obtain a similar result, although without making explicit the dependence of the constant of the estimate on $|w-y|$.

TheOREM 4.4. Let $y \in \Omega$ and $G^{y} \in L^{2}(\Omega)$ be the solution of problem (3.6). Let $h_{0}>0$ be such that $\forall h \in\left(0, h_{0}\right]$ problem (4.2) has a unique solution $G_{h}^{y}$. Given $w \in \Omega$, $w \neq y$, let $d>0$ be such that $|w-y| \geq d$, $\operatorname{dist}(w, \partial \Omega) \geq d$, and $\operatorname{dist}(y, \partial \Omega) \geq d$. Then, there exist strictly positive constants $h_{1}<h_{0}$ and $C$, both depending on $d$, such that, $\forall h \in\left(0, h_{1}\right]$,

$$
\left|G^{y}(w)-G_{h}^{y}(w)\right| \leq C h^{2} \ln \left(\frac{1}{h}\right)
$$

Proof. The proof is essentially identical to that of Theorem 6.1(i) of [13]. Thus, we only include here its main steps and emphasize those which differ in our case.

Let $D_{1}:=\mathcal{B}_{d / 4}(w), D_{2}:=\mathcal{B}_{d / 2}(w)$ and $D^{\prime}:=\mathcal{B}_{d / 4}(y)$. Then $\operatorname{dist}\left(D_{1}, D^{\prime}\right) \geq \frac{d}{2}$. Applying Theorem 5.1 in [13] we know that there exist constants $C>0$ and $h_{1}>0$, both depending on $d$, such that $\forall h \in\left(0, h_{1}\right]$ and $\forall q_{h} \in \mathcal{V}_{h}$,

$$
\left|G^{y}(w)-G_{h}^{y}(w)\right| \leq C \ln \left(\frac{1}{h}\right)\left[\left\|G^{y}-q_{h}\right\|_{L^{\infty}\left(D_{1}\right)}+\left\|G^{y}-G_{h}^{y}\right\|_{L^{1}\left(D_{1}\right)}\right] .
$$

The first term in the right-hand side can be bounded by using Lemma 3.4 (Part 1) and the standard error estimate for the Lagrange interpolation (see for instance [2]); namely, if $h<d / 4$, then

$$
\inf _{q_{h} \in \mathcal{V}_{h}}\left\|G^{y}-q_{h}\right\|_{L^{\infty}\left(D_{1}\right)} \leq C h^{2}\left\|G^{y}\right\|_{W^{2, \infty}\left(D_{2}\right)} \leq C h^{2} .
$$

For the second term, we apply the same duality argument as in the proof of Theorem 6.1(i) of [13]. By doing so, we can repeat all the steps of this proof with the exception of the following one: Given $q \in \mathcal{C}_{0}^{\infty}\left(D_{1}\right)$, let

$$
r \in W^{1, \infty}(\Omega): \quad a_{\omega}(s, r)=\int_{\Omega} s \bar{q} d x, \quad \forall s \in W^{1,1}(\Omega) ;
$$

it has to be proved that $\|r\|_{W^{2, \infty}\left(D^{\prime}\right)} \leq C\|q\|_{L^{\infty}\left(D_{1}\right)}$. We do it in our case by repeating the arguments in the proof of Lemma 3.4 (Part 2).

The rest of the proof runs essentially as that of Theorem 6.1(i) of [13].

5. Optimal amplitudes of actuators. Numerical methods. From now on we assume that the primary source $g$ is such that $\left.g\right|_{\Gamma_{\mathrm{N}}^{j}} \in H^{1 / 2}\left(\Gamma_{\mathrm{N}}^{j}\right), j=1, \ldots, J$, and the secondary source is given by (2.2) in terms of the control variable $\boldsymbol{u}=$ $\left(u_{1}, \ldots, u_{N}\right) \in \mathbb{C}^{N}: f:=\sum_{i=1}^{N} u_{i} \delta_{y_{i}}$, with $y_{i} \in \Omega, i=1, \ldots, N$.

Let $p$ be the solution of problem (2.1) with such $f$. Due to the linearity of the Helmholtz equation $p$ can be written in terms of the control variable as follows:

$$
p=p_{0}+\sum_{i=1}^{N} u_{i} G^{y_{i}},
$$


where $p_{0}$ is the pressure field arising from the primary source $g$ without any control; more precisely, $p_{0}$ is the solution of problem (2.1) for $\boldsymbol{u}=\mathbf{0}$ (i.e., $f=0$ ). In its turn, $G^{y_{i}}$ is the solution of problem (3.6) with $y=y_{i}, i=1, \ldots, N$. This corresponds to the pressure field when the system is only excited by the $i^{\text {th }}$ loud-speaker with unit amplitude, excluding the effect of the primary source. Note that according to Theorem 3.3 and (3.11), it makes sense to evaluate $p$ at points $w \in \Omega, w \neq y_{i}$, $i=1, \ldots, N$.

Let $w_{1}, \ldots, w_{M} \in \Omega$ be such that $\left\{y_{1}, \ldots, y_{N}\right\}$ and $\left\{w_{1}, \ldots, w_{M}\right\}$ are disjoint. To prove existence of an optimal control is an easy task because the control space is finite-dimensional. It relies upon the fact that the mapping giving the observation from the control, namely,

$$
\begin{aligned}
& \mathbb{C}^{N} \longrightarrow \mathbb{C}^{M} \\
& \boldsymbol{u} \longmapsto \boldsymbol{z}(\boldsymbol{u})=\left(p\left(w_{i}\right), \ldots, p\left(w_{M}\right)\right)
\end{aligned}
$$

is affine (and then continuous). The mapping $\boldsymbol{z}(\boldsymbol{u})$ is the so-called transfer function, which establishes the relation between controls and observations.

Therefore, the cost function (2.3) is quadratic. The first term of the cost function is convex since the observation $\boldsymbol{z}(\boldsymbol{u})$ is affine and the second one is strictly convex when $\nu>0$. Therefore, it is clear that the function $J$ is strictly convex under any of the two following assumptions:

- $\nu>0$,

- $\nu \geq 0$ and $\boldsymbol{z}(\boldsymbol{u})$ is one-to-one,

in which case there exists a unique optimal control.

We notice that $\boldsymbol{z}(\boldsymbol{u})$ is one-to-one if and only if the observations corresponding to each single actuator are linearly independent. Obviously this can happen only if the number of microphones is greater or equal than the number of loud-speakers: $M \geq N$.

To write the control problem in matrix form, we introduce the vectors,

$$
\begin{aligned}
& \boldsymbol{z}_{0}:=\left(p_{0}\left(w_{1}\right), \ldots, p_{0}\left(w_{M}\right)\right) \in \mathbb{C}^{M}, \\
& \boldsymbol{z}_{i}:=\left(G^{y_{i}}\left(w_{1}\right), \ldots, G^{y_{i}}\left(w_{M}\right)\right) \in \mathbb{C}^{M}, \quad i=1, \ldots, N .
\end{aligned}
$$

Note that according to Theorem 3.3 and (3.11), respectively, there hold

$$
\left\|\boldsymbol{z}_{0}\right\| \leq C \sum_{j=1}^{J}\|g\|_{H^{1 / 2}\left(\Gamma_{\mathrm{N}}^{j}\right)} \quad \text { and } \quad\left\|\boldsymbol{z}_{i}\right\| \leq C, \quad i=1, \ldots, N .
$$

The observation $\boldsymbol{z}$ can be written in terms of the control variable $\boldsymbol{u} \in \mathbb{C}^{N}$ and the observations $\boldsymbol{z}_{0}, \boldsymbol{z}_{1}, \ldots, \boldsymbol{z}_{N}$ in the following way:

$$
\boldsymbol{z}(\boldsymbol{u})=\boldsymbol{z}_{0}+\sum_{i=1}^{N} u_{i} \boldsymbol{z}_{i}
$$

Then, the cost function becomes

$$
\begin{aligned}
J(\boldsymbol{u}) & =\frac{1}{2}\left\|\boldsymbol{z}_{0}+\sum_{i=1}^{N} u_{i} \boldsymbol{z}_{i}\right\|^{2}+\frac{\nu}{2}\|\boldsymbol{u}\|^{2} \\
& =\frac{1}{2}\left[\overline{\boldsymbol{z}}_{0}^{\mathrm{t}} \boldsymbol{z}_{0}+2 \operatorname{Re}\left(\sum_{i=1}^{N} \bar{u}_{i} \overline{\boldsymbol{z}}_{i}^{\mathrm{t}} \boldsymbol{z}_{0}\right)+\sum_{i, j=1}^{N} u_{j} \bar{u}_{i}\left(\overline{\boldsymbol{z}}_{i}^{\mathrm{t}} \boldsymbol{z}_{j}+\nu \delta_{i j}\right)\right] .
\end{aligned}
$$


Let us define the matrix $\boldsymbol{Z} \in \mathbb{C}^{N \times N}$ and the vector $\boldsymbol{d} \in \mathbb{C}^{N}$ by

$$
\begin{gathered}
(\boldsymbol{Z})_{i j}:=\overline{\boldsymbol{z}}_{i}^{\mathrm{t}} \boldsymbol{z}_{j}, \quad i, j=1, \ldots, N, \\
(\boldsymbol{d})_{i}:=\overline{\boldsymbol{z}}_{i}^{\mathrm{t}} \boldsymbol{z}_{0}, \quad i=1, \ldots, N .
\end{gathered}
$$

Then, the optimal control problem (2.4) is equivalent to the following quadratic programming problem:

Find $\boldsymbol{u}^{\mathrm{op}} \in U_{\mathrm{ad}}$ such that

$$
J\left(\boldsymbol{u}^{\mathrm{op}}\right)=\inf _{\boldsymbol{u} \in U_{\mathrm{ad}}} \frac{1}{2}\left[\overline{\boldsymbol{u}}^{\mathrm{t}}(\boldsymbol{Z}+\nu \boldsymbol{I}) \boldsymbol{u}+2 \operatorname{Re}\left(\overline{\boldsymbol{u}}^{\mathrm{t}} \boldsymbol{d}\right)+\left\|\boldsymbol{z}_{0}\right\|^{2}\right] .
$$

Although the cost function is defined in a finite dimensional space, it involves the solution of a partial differential equation which has to be approximated by means of some discretization process as, for instance, the finite element method described in Section 4. This leads to approximate observations and thereby to an approximate cost function.

Similar definitions hold for the approximate observations. Given $\boldsymbol{u} \in \mathbb{C}^{N}$, let

$$
\boldsymbol{z}_{h}(\boldsymbol{u}):=\left(p_{h}\left(w_{i}\right), \ldots, p_{h}\left(w_{M}\right)\right) \in \mathbb{C}^{M} .
$$

where $p_{h}$ is the solution of the discrete problem (4.1) with $f$ defined by (2.2) as above. Let $\boldsymbol{z}_{i h} \in \mathbb{C}^{M}$ be defined by

$$
\begin{aligned}
\boldsymbol{z}_{0 h} & :=\left(p_{0 h}\left(w_{1}\right), \ldots, p_{0 h}\left(w_{M}\right)\right) \in \mathbb{C}^{M}, \\
\boldsymbol{z}_{i h} & :=\left(G_{h}^{y_{i}}\left(w_{1}\right), \ldots, G_{h}^{y_{i}}\left(w_{M}\right)\right) \in \mathbb{C}^{M}, \quad i=1, \ldots, N,
\end{aligned}
$$

where $p_{0 h}$ is the solution of problem (4.1) for $\boldsymbol{u}=\mathbf{0}$ (i.e., $f=0$ ), and $G_{h}^{y_{i}}$ is the solution of the discrete problem (4.2) for $y=y_{i}, i=1, \ldots, N$. Then, $\boldsymbol{z}_{h}(\boldsymbol{u}) \in \mathbb{C}^{M}$ is given by

$$
\boldsymbol{z}_{h}(\boldsymbol{u}):=\boldsymbol{z}_{0 h}+\sum_{i=1}^{N} u_{i} \boldsymbol{z}_{i h} .
$$

Let $\boldsymbol{Z}_{h} \in \mathbb{C}^{N \times N}$ and $\boldsymbol{d}_{h} \in \mathbb{C}^{N}$ be defined by

$$
\begin{aligned}
\left(\boldsymbol{Z}_{h}\right)_{i j} & :=\overline{\boldsymbol{z}}_{i h}^{\mathrm{t}} \boldsymbol{z}_{j h}, \quad i, j=1, \ldots, N, \\
\left(\boldsymbol{d}_{h}\right)_{i} & :=\overline{\boldsymbol{z}}_{i h}^{\mathrm{t}} \boldsymbol{z}_{0 h}, \quad i=1, \ldots, N .
\end{aligned}
$$

Then the approximate cost function can be written

$$
J_{h}(\boldsymbol{u}):=\frac{1}{2}\left\|\boldsymbol{z}_{h}(\boldsymbol{u})\right\|^{2}+\frac{\nu}{2}\|\boldsymbol{u}\|^{2}=\frac{1}{2}\left[\overline{\boldsymbol{u}}^{\mathrm{t}}\left(\boldsymbol{Z}_{h}+\nu \boldsymbol{I}\right) \boldsymbol{u}+2 \operatorname{Re}\left(\overline{\boldsymbol{u}}^{\mathrm{t}} \boldsymbol{d}_{h}\right)+\left\|\boldsymbol{z}_{0 h}\right\|^{2}\right] .
$$

These definitions lead us to the following discrete optimal control problem:

Find $\boldsymbol{u}_{h}^{\text {op }} \in U_{\text {ad }}$ such that

$$
J_{h}\left(\boldsymbol{u}_{h}^{\mathrm{op}}\right)=\inf _{\boldsymbol{u} \in U_{\mathrm{ad}}} \frac{1}{2}\left[\overline{\boldsymbol{u}}^{\mathrm{t}}\left(\boldsymbol{Z}_{h}+\nu \boldsymbol{I}\right) \boldsymbol{u}+2 \operatorname{Re}\left(\overline{\boldsymbol{u}}^{\mathrm{t}} \boldsymbol{d}_{h}\right)+\left\|\boldsymbol{z}_{0 h}\right\|^{2}\right] .
$$

The argument $\boldsymbol{u}_{h}^{\text {op }}$ where the minimum is attained is expected to be an approximation of the optimal control $\boldsymbol{u}^{\mathrm{op}}$. Our next goal is to obtain an estimate for this approximation. To this aim, we denote

$$
\delta \boldsymbol{d}:=\boldsymbol{d}-\boldsymbol{d}_{h} \quad \text { and } \quad \delta \boldsymbol{Z}:=\boldsymbol{Z}-\boldsymbol{Z}_{h} .
$$


Lemma 5.1. There exists strictly positive constants $C$ and $h_{0}$ such that for all $h \in\left(0, h_{0}\right]$ the following inequalities hold:

$$
\|\delta \boldsymbol{d}\| \leq C h^{2} \ln \left(\frac{1}{h}\right) \sum_{j=1}^{J}\|g\|_{H^{1 / 2}\left(\Gamma_{\mathrm{N}}^{j}\right)}, \quad\|\delta \boldsymbol{Z}\| \leq C h^{2} \ln \left(\frac{1}{h}\right) .
$$

Moreover, if $\boldsymbol{Z}$ is positive definite, then so is $\boldsymbol{Z}_{h}$ for $h$ small enough.

Proof. First we settle an error estimate for the observations $\boldsymbol{z}_{0}, \boldsymbol{z}_{1}, \ldots, \boldsymbol{z}_{N}$. We denote the corresponding errors by

$$
\delta \boldsymbol{z}_{i}:=\boldsymbol{z}_{i h}-\boldsymbol{z}_{i}, \quad i=0, \ldots, N .
$$

From Theorem 4.2, for $h$ small enough we have

$$
\left\|\delta \boldsymbol{z}_{0}\right\|=\left[\sum_{k=1}^{M}\left|p_{0}\left(w_{k}\right)-p_{0 h}\left(w_{k}\right)\right|^{2}\right]^{1 / 2} \leq C h^{2} \ln \left(\frac{1}{h}\right) \sum_{j=1}^{J}\|g\|_{H^{1 / 2}\left(\Gamma_{\mathrm{N}}^{j}\right)},
$$

whereas from Theorem 4.4,

$$
\left\|\delta \boldsymbol{z}_{i}\right\|=\left[\sum_{k=1}^{M}\left|G^{y_{i}}\left(w_{k}\right)-G_{h}^{y_{i}}\left(w_{k}\right)\right|^{2}\right]^{1 / 2} \leq C h^{2} \ln \left(\frac{1}{h}\right) .
$$

Therefore, if $h$ is small enough,

$$
\begin{aligned}
\|\delta \boldsymbol{d}\| & =\left[\sum_{i=1}^{N}\left|\overline{\boldsymbol{z}}_{0}^{\mathrm{t}} \boldsymbol{z}_{i}-\left(\overline{\boldsymbol{z}}_{0}+\delta \overline{\boldsymbol{z}}_{0}\right)^{\mathrm{t}}\left(\boldsymbol{z}_{i}+\delta \boldsymbol{z}_{i}\right)\right|^{2}\right]^{1 / 2} \\
& \leq\left[\sum_{i=1}^{N}\left(\left\|\boldsymbol{z}_{0}\right\|\left\|\delta \boldsymbol{z}_{i}\right\|+\left\|\delta \boldsymbol{z}_{0}\right\|\left\|\boldsymbol{z}_{i}\right\|+\left\|\delta \boldsymbol{z}_{0}\right\|\left\|\delta \boldsymbol{z}_{i}\right\|\right)^{2}\right]^{1 / 2} \\
& \leq C h^{2} \ln \left(\frac{1}{h}\right) \sum_{j=1}^{J}\|g\|_{H^{1 / 2}\left(\Gamma_{N}^{j}\right)}
\end{aligned}
$$

the last inequality because of (5.2).

The error bound for $\|\delta \boldsymbol{Z}\|$ is proved essentially in the same way. Furthermore, since $\boldsymbol{Z}_{h}$ converges to $\boldsymbol{Z}$, if $\boldsymbol{Z}$ is positive definite, then for $h$ small enough $\boldsymbol{Z}_{h}$ is positive definite too.

As an immediate consequence of the above lemma we have existence and uniqueness of solution of the discrete optimal control problem, for $h$ sufficiently small.

Corollary 5.2. Let us assume that $\nu>0$ or $\nu \geq 0$ and $\boldsymbol{z}(\boldsymbol{u})$ is one-to-one. Then, there exists $h_{0}>0$ such that, for all $h \in\left(0, h_{0}\right]$, problem (5.3) has a unique solution.

To obtain a bound for $\left\|\boldsymbol{u}^{\mathrm{op}}-\boldsymbol{u}_{h}^{\mathrm{op}}\right\|$, we prove first the following a priori error estimate for the solution of a variational inequality subject to data perturbations.

Lemma 5.3. Let $U_{\text {ad }}$ be a convex subset of $\mathbb{C}^{N}, \boldsymbol{b} \in \mathbb{C}^{N}$, and $\boldsymbol{A} \in \mathbb{C}^{N \times N}$ a positive definite Hermitian matrix. Let $\alpha>0$ be such that

$$
\overline{\boldsymbol{v}}^{\mathrm{t}} \boldsymbol{A} \boldsymbol{v} \geq \alpha\|\boldsymbol{v}\|^{2} \quad \forall \boldsymbol{v} \in \mathbb{C}^{N}
$$


Let $\delta \boldsymbol{b} \in \mathbb{C}^{N}$ and $\delta \boldsymbol{A} \in \mathbb{C}^{N \times N}$ be such that $\|\delta \boldsymbol{A}\|<\alpha$. Let $\boldsymbol{u} \in U_{\mathrm{ad}}$ and $(\boldsymbol{u}+\delta \boldsymbol{u}) \in U_{\mathrm{ad}}$ be the solutions of the following variational inequalities:

$$
\begin{aligned}
& \operatorname{Re}\left[(\overline{\boldsymbol{v}}-\overline{\boldsymbol{u}})^{\mathrm{t}}(\boldsymbol{A} \boldsymbol{u}+\boldsymbol{b})\right] \geq 0 \quad \forall \boldsymbol{v} \in U_{\mathrm{ad}}, \\
& \operatorname{Re}\left\{[\overline{\boldsymbol{v}}-(\overline{\boldsymbol{u}}+\delta \bar{u})]^{\mathrm{t}}[(\boldsymbol{A}+\delta \boldsymbol{A})(\boldsymbol{u}+\delta \boldsymbol{u})+(\boldsymbol{b}+\delta \boldsymbol{b})]\right\} \geq 0 \quad \forall \boldsymbol{v} \in U_{\mathrm{ad}} .
\end{aligned}
$$

Then

$$
\|\delta \boldsymbol{u}\| \leq \frac{1}{\alpha-\|\delta \boldsymbol{A}\|}(\|\delta \boldsymbol{A}\|\|\boldsymbol{u}\|+\|\delta \boldsymbol{b}\|) .
$$

Furthermore, if $U_{\mathrm{ad}} \ni \mathbf{0}$ and $\|\delta \boldsymbol{A}\|<\theta \alpha$ with $0<\theta<1$, then

$$
\|\delta \boldsymbol{u}\| \leq \frac{1}{(1-\theta) \alpha}\left(\frac{\|\boldsymbol{b}\|}{\alpha}\|\delta \boldsymbol{A}\|+\|\delta \boldsymbol{b}\|\right) .
$$

Proof. By taking $\boldsymbol{v}=\boldsymbol{u}+\delta \boldsymbol{u}$ in (5.4) and $\boldsymbol{v}=\boldsymbol{u}$ in (5.5) we obtain

$$
\begin{aligned}
& \operatorname{Re}\left[\delta \overline{\boldsymbol{u}}^{\mathrm{t}}(\boldsymbol{A} \boldsymbol{u}+\boldsymbol{b})\right] \geq 0, \\
& \operatorname{Re}\left\{-\delta \overline{\boldsymbol{u}}^{\mathrm{t}}[(\boldsymbol{A}+\delta \boldsymbol{A})(\boldsymbol{u}+\delta \boldsymbol{u})+(\boldsymbol{b}+\delta \boldsymbol{b})]\right\} \geq 0 .
\end{aligned}
$$

By adding these inequalities we obtain

$$
\operatorname{Re}\left(\overline{\delta \boldsymbol{u}}^{\mathrm{t}} \boldsymbol{A} \delta \boldsymbol{u}\right) \leq \operatorname{Re}\left\{-\overline{\delta \boldsymbol{u}}^{\mathrm{t}}[\delta \boldsymbol{A}(\boldsymbol{u}+\delta \boldsymbol{u})+\delta \boldsymbol{b}]\right\} .
$$

Then, since $\boldsymbol{A}$ is Hermitian and positive definite we have

$$
\alpha\|\delta \boldsymbol{u}\|^{2} \leq \overline{\delta \boldsymbol{u}}^{\mathrm{t}} \boldsymbol{A} \delta \boldsymbol{u}=\operatorname{Re}\left(\overline{\delta \boldsymbol{u}}^{\mathrm{t}} \boldsymbol{A} \delta \boldsymbol{u}\right) \leq\|\delta \boldsymbol{u}\|(\|\delta \boldsymbol{A}\|\|\delta \boldsymbol{u}\|+\|\delta \boldsymbol{A}\|\|\boldsymbol{u}\|+\|\delta \boldsymbol{b}\|),
$$

and, therefore,

$$
(\alpha-\|\delta \boldsymbol{A}\|)\|\delta \boldsymbol{u}\| \leq\|\delta \boldsymbol{A}\|\|\boldsymbol{u}\|+\|\delta \boldsymbol{b}\| .
$$

Hence, for $\|\delta \boldsymbol{A}\|<\alpha$, we obtain (5.6). Moreover, if $\|\delta \boldsymbol{A}\|<\theta \alpha$ with $0<\theta<1$, then

$$
\|\delta \boldsymbol{u}\| \leq \frac{1}{(1-\theta) \alpha}(\|\delta \boldsymbol{A}\|\|\boldsymbol{u}\|+\|\delta \boldsymbol{b}\|) .
$$

On the other hand, if $U_{\text {ad }} \ni \mathbf{0}$, then we can take $\boldsymbol{v}=\mathbf{0}$ in (5.4) and we obtain $\operatorname{Re}\left[-\overline{\boldsymbol{u}}^{\mathrm{t}}(\boldsymbol{A u}+\boldsymbol{b})\right] \geq 0$. Then

$$
\alpha\|\delta \boldsymbol{u}\|^{2} \leq \overline{\boldsymbol{u}}^{\mathrm{t}} \boldsymbol{A} \boldsymbol{u}=\operatorname{Re}\left(\overline{\boldsymbol{u}}^{\mathrm{t}} \boldsymbol{A} \boldsymbol{u}\right) \leq \operatorname{Re}\left(-\overline{\boldsymbol{u}}^{\mathrm{t}} \boldsymbol{b}\right) \leq\|\delta \boldsymbol{u}\|\|\delta \boldsymbol{b}\| .
$$

Hence, $\|\delta \boldsymbol{u}\| \leq\|\delta \boldsymbol{b}\| / \alpha$, and (5.7) follows from this inequality and (5.8). Thus we conclude the proof.

From the above lemma and the error estimates of Lemma 5.1 it is easy to prove the following result.

THEOREM 5.4. Let us assume that $\nu>0$ or $\nu \geq 0$ and $\boldsymbol{z}(\boldsymbol{u})$ is one-to-one. If $U_{\text {ad }} \ni \mathbf{0}$, then there exist $C>0$ and $h_{0}>0$ such that, for all $h \in\left(0, h_{0}\right]$,

$$
\left\|\boldsymbol{u}^{\mathrm{op}}-\boldsymbol{u}_{h}^{\mathrm{op}}\right\| \leq C h^{2} \ln \left(\frac{1}{h}\right) \sum_{j=1}^{J}\|g\|_{H^{1 / 2}\left(\Gamma_{\mathrm{N}}^{j}\right)} .
$$


Proof. Let $\delta \boldsymbol{u}:=\boldsymbol{u}_{h}^{\mathrm{op}}-\boldsymbol{u}^{\mathrm{op}}$. The exact and the approximate optimal controls satisfy the variational inequalities

$$
\begin{aligned}
& \operatorname{Re}\left\{\left(\overline{\boldsymbol{v}}-\overline{\boldsymbol{u}}^{\mathrm{op}}\right)^{\mathrm{t}}\left[(\boldsymbol{Z}+\nu \boldsymbol{I}) \boldsymbol{u}^{\mathrm{op}}+\boldsymbol{d}\right]\right\} \geq 0 \quad \forall \boldsymbol{v} \in U_{\mathrm{ad}}, \\
& \operatorname{Re}\left\{\left(\boldsymbol{v}-\boldsymbol{u}_{h}^{\mathrm{op}}\right)^{\mathrm{t}}\left[(\boldsymbol{Z}+\nu \boldsymbol{I}+\delta \boldsymbol{Z})\left(\boldsymbol{u}^{\mathrm{op}}+\delta \boldsymbol{u}\right)+(\boldsymbol{d}+\delta \boldsymbol{d})\right]\right\} \geq 0 \quad \forall \boldsymbol{v} \in U_{\mathrm{ad}} .
\end{aligned}
$$

Since $\boldsymbol{Z}+\nu \boldsymbol{I}$ is a Hermitian positive definite matrix, let $\alpha>0$ be such that

$$
\overline{\boldsymbol{v}}^{\mathrm{t}}(\boldsymbol{Z}+\nu \boldsymbol{I}) \boldsymbol{v} \geq \alpha\|\boldsymbol{v}\|^{2} \quad \forall \boldsymbol{v} \in U_{\mathrm{ad}} .
$$

According to Lemma 5.1, for $h$ sufficiently small $\|\delta \boldsymbol{Z}\|<\alpha / 2$. Then, we can apply Lemma 5.3 to the variational inequalities above and we obtain

$$
\left\|\boldsymbol{u}^{\mathrm{op}}-\boldsymbol{u}_{h}^{\mathrm{op}}\right\| \leq \frac{2}{\alpha}\left(\frac{\|\boldsymbol{d}\|}{\alpha}\|\delta \boldsymbol{Z}\|+\|\delta \boldsymbol{d}\|\right) .
$$

Thus, we conclude the proof from this inequality, (5.2), and Lemma 5.1.

REMARK 2. The assumption made on the admissible set, $U_{\mathrm{ad}} \ni \mathbf{0}$, to prove the error estimate of this theorem is not restrictive at all in practice. It just means that a vanishing control is also admissible.

6. Numerical results. In this section we present some numerical results for a $3 \mathrm{D}$ test. In order to assess the effect of the control we use the following measure of attenuation:

$$
\text { Attenuation }(\mathrm{dB})=-10 \log _{10}\left[\frac{J\left(\boldsymbol{u}^{\mathrm{op}}\right)}{J(\mathbf{0})}\right] .
$$

The data of the test are the following:

- the domain is $\Omega=[0,1] \mathrm{m} \times[0,1] \mathrm{m} \times[0,1] \mathrm{m}$;

- the physical parameters are $\omega=680 \mathrm{~s}^{-1}, c=340 \mathrm{~m} \mathrm{~s}^{-1}$, and $\rho=1 \mathrm{~kg} \mathrm{~m}^{-3}$;

- the amplitude of the primary source of noise is $g(x, y, 0)=\mathrm{e}^{i y} \mathrm{~kg} \mathrm{~m}^{-2} \mathrm{~s}^{-2}$ on the wall $z=0$;

- there is one loud-speaker located at $y_{1}=\left(\frac{4}{6}, \frac{4}{6}, \frac{4}{6}\right) \mathrm{m}$;

- there are two microphones at $w_{1}=\left(\frac{1}{6}, \frac{2}{6}, \frac{1}{6}\right) \mathrm{m}$ and $w_{2}=\left(\frac{5}{6}, \frac{1}{6}, \frac{3}{6}\right) \mathrm{m}$;

- on the wall $z=1$, the acoustic impedance is $Z=(102+340 i) \times 10^{3} \mathrm{~kg} \mathrm{~m}^{-2} \mathrm{~s}^{-1}$;

- the rest of the walls are perfectly rigid;

- the admissible set of controls is $U_{\mathrm{ad}}=\mathbb{C}$ and the weighting factor is $\nu=0$.

The optimal control has been computed for several meshes which have been obtained by uniformly refining the coarse mesh shown in Figure 6.1.

Then, a more accurate value of the optimal control has been determined by extrapolating the controls computed on these meshes. This more accurate value has been used to compute the relative errors of the real and imaginary parts of the computed controls. These errors are shown in Figure 6.2, where it can be clearly observed that the order of convergence is essentially $\mathcal{O}\left(h^{2}\right)$ as predicted by the theoretical results.

For $h=1 / 24$, the computed attenuation is $0.75 \mathrm{~dB}$. The modulus of the complex pressure fields on the plane containing the actuator and the two sensors are shown in Figures 6.3 and 6.4. The first one corresponds to the system without control, whereas the second one shows the pressure with the optimal control. 


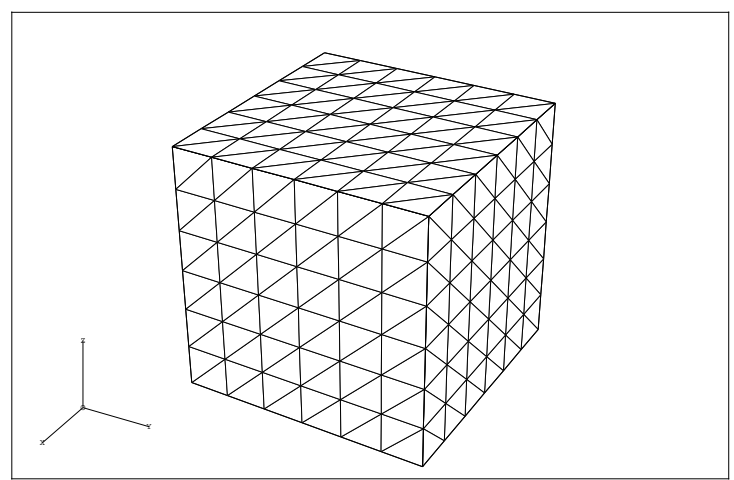

FIG. 6.1. Coarsest mesh $(h=1 / 6 \mathrm{~m})$.

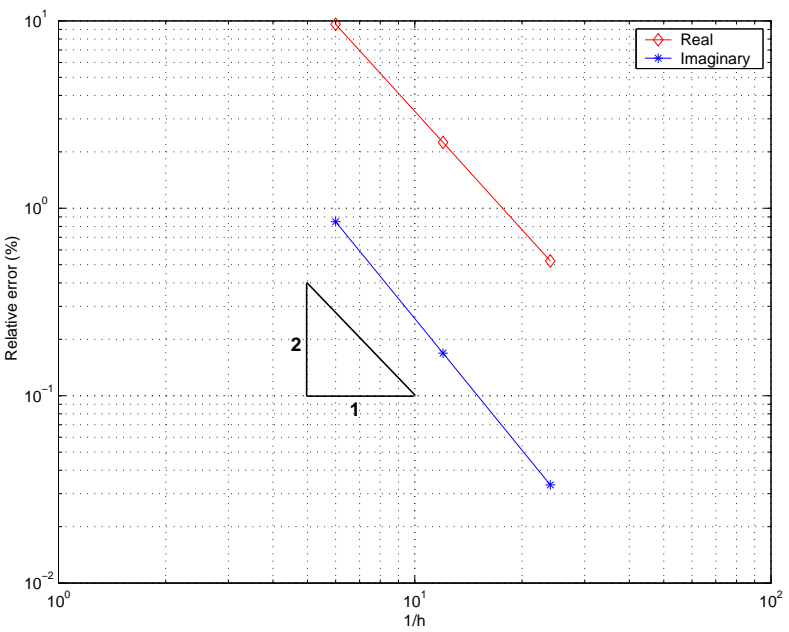

FIG. 6.2. Relative error (\%) as a function of $1 / h$ in log-log scale.

Finally Figure 6.5 shows the local attenuation field computed on the same plane: $\operatorname{Att}(w)=-10 \log _{10}\left[\left|p_{h}(w)\right|^{2} /\left|p_{0 h}(w)\right|^{2}\right]$.

In this case, it can be observed that there exist zones where the noise is reinforced; that is, where primary and secondary sources interfere in a constructive way. This happens for instance in the location of the first sensor. Indeed, the noise level without control is low around this sensor and too high around the other one (see Figure 6.3). Thus, to obtain a minimum of the cost functional, the optimal amplitude of the actuator is such that it produces a noise reinforcement in the first sensor. Anyway, the comparison of Figures 6.3 and 6.4 shows that the global attenuation has been significant in the whole domain, except for the vicinity of the actuator.

7. Optimal location of actuators. In the previous sections the position of both, sensors (microphones) and actuators (loud-speakers), were given. Then we have used the complex amplitudes of the actuators as the unique control variable and have determined their optimal values with the objective of minimizing the pressure level 


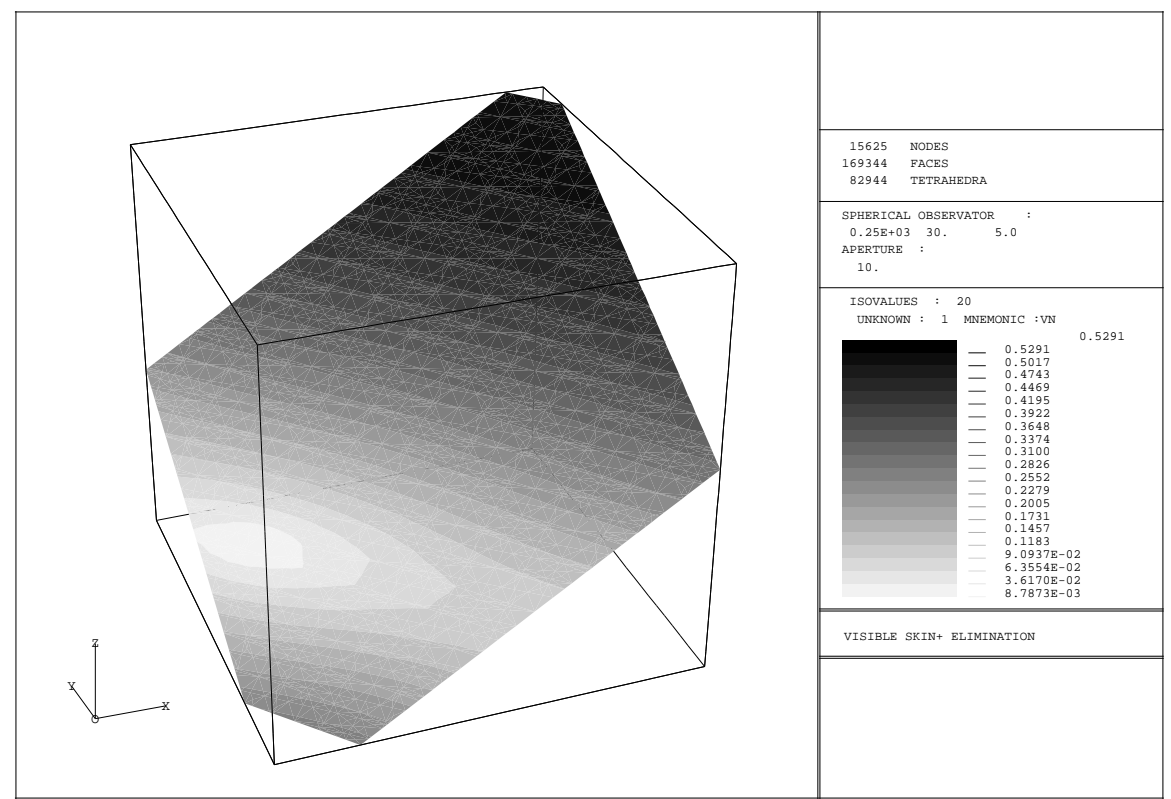

FIG. 6.3. Modulus of the pressure field without control $(h=1 / 24 \mathrm{~m})$.

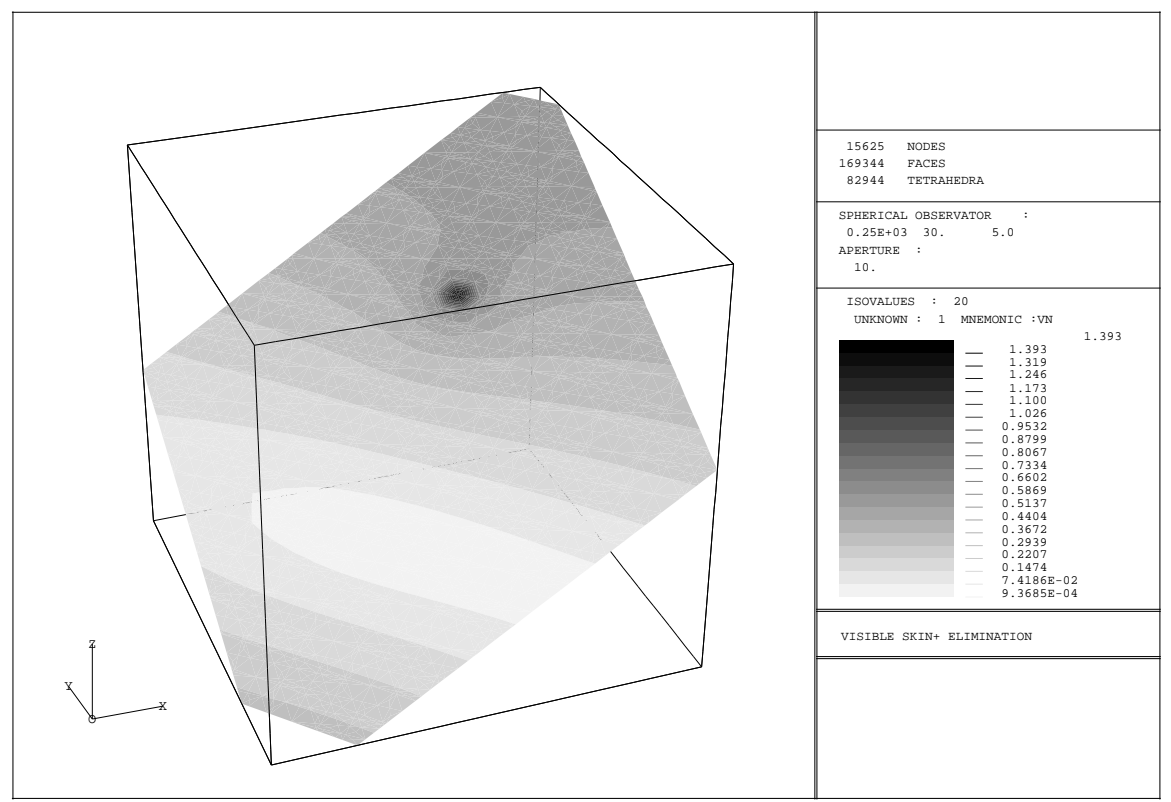

Fig. 6.4. Modulus of the pressure field with control $(h=1 / 24 \mathrm{~m})$.

at those points where the sensors were located. Now we assume that the positions of the actuators can also be chosen in certain subsets of the domain and we will try to determine those that minimize the same objective function as above, when the complex amplitudes are optimal with respect to these positions. This is the most important problem when a system of active control of sound has to be implemented to reduce noise in an enclosure. It can also be formulated as an optimal control 


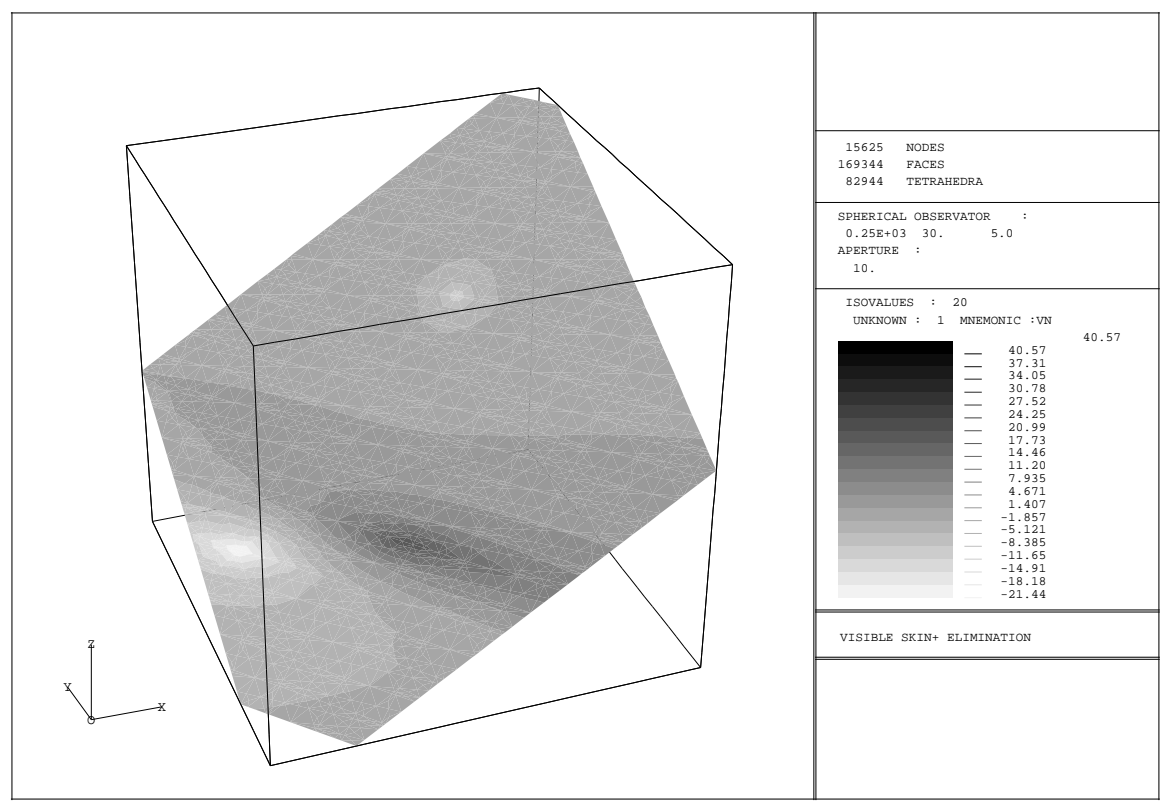

FIG. 6.5. Attenuation field $(h=1 / 24 \mathrm{~m})$.

problem.

In this case the control variables are the complex amplitudes (modulus and phases) and the positions of the actuators,

$$
\boldsymbol{u}=\left(u_{1}, \ldots, u_{N}\right) \in \mathbb{C}^{N} \text { and } \boldsymbol{y}=\left(y_{1}, \ldots, y_{N}\right) \in \Omega^{N},
$$

respectively, which define the secondary source by means of (2.2).

We consider the set of admissible controls $U_{\text {ad }} \times Y_{\text {ad }} \subset \mathbb{C}^{N} \times \Omega^{N}$, where $U_{\text {ad }} \subset \mathbb{C}^{N}$ and $Y_{\text {ad }} \subset \subset\left(\Omega \backslash\left\{w_{1}, \ldots, w_{M}\right\}\right)^{N}$ are closed convex subsets.

The observation $\boldsymbol{z}(\boldsymbol{u}, \boldsymbol{y})$ is again the set of pressure values at the microphones locations $w_{1}, \ldots, w_{M} \in \Omega$. The transfer function is now:

$$
\begin{array}{clc}
U_{\mathrm{ad}} \times Y_{\mathrm{ad}} & \longrightarrow & \mathbb{C}^{M} \\
(\boldsymbol{u}, \boldsymbol{y}) & \longmapsto & \boldsymbol{z}(\boldsymbol{u}, \boldsymbol{y})=\left(p\left(w_{i}\right), \ldots, p\left(w_{M}\right)\right)
\end{array}
$$

where, for each admissible set of values of the control variables, $\boldsymbol{u} \in U_{\mathrm{ad}}$ and $\boldsymbol{y} \in Y_{\mathrm{ad}}$, $p$ denotes again the solution of the state equation (2.1) with $f$ given by (2.2). Notice that the sensors locations are excluded of the domain of admissible locations for the actuators, to ensure the continuity of the transfer function.

The cost function is given again by

$$
J(\boldsymbol{u}, \boldsymbol{y}):=\frac{1}{2}\|\boldsymbol{z}(\boldsymbol{u}, \boldsymbol{y})\|^{2}+\frac{\nu}{2}\|\boldsymbol{u}\|^{2}
$$

and, then, the optimal control problem is as follows:

Find $\left(\boldsymbol{u}^{\mathrm{op}}, \boldsymbol{y}^{\mathrm{op}}\right) \in U_{\mathrm{ad}} \times Y_{\text {ad }}$ such that

$$
J\left(\boldsymbol{u}^{\mathrm{op}}, \boldsymbol{y}^{\mathrm{op}}\right)=\inf _{(\boldsymbol{u}, \boldsymbol{y}) \in U_{\mathrm{ad}} \times Y_{\mathrm{ad}}} J(\boldsymbol{u}, \boldsymbol{y}) .
$$


The difficulty now is that the dependency of the state with respect to the additional control variables (the positions of actuators) is no longer affine. Thus the cost function may have many local minima and therefore gradient-like methods are not suitable to solve the problem. In practice, the number of feasible locations is typically finite and hence the optimization problem becomes an integer programming problem. In these cases one can use, for instance, genetic or simulated annealing algorithms. Once the optimal locations have been determined it will be possible to improve them in some given neighborhoods by performing a local minimization using the gradient of the cost function with respect to the location of the actuators. As we will see below the first order optimality conditions allow computing the gradient of the cost function through an adjoint state.

7.1. Existence of an optimal control. Optimality conditions. We recall that the pressure field defining the observations can be written in terms of the control variables by means of (5.1): $p=p_{0}+\sum_{i=1}^{N} u_{i} G^{y_{i}}$. Then, the transfer function is affine with respect to $\boldsymbol{u}$ although it is nonlinear with respect to $\boldsymbol{y}$.

Therefore, the optimal control problem (7.1) has a solution as a direct consequence of the following facts:

- the function $J(\boldsymbol{u}, \boldsymbol{y})$ is continuous in $U_{\mathrm{ad}} \times Y_{\mathrm{ad}}$,

- the set $Y_{\text {ad }}$ is compact,

- $U_{\text {ad }}$ is a closed set, and

- $J(\boldsymbol{u}, \boldsymbol{y}) \rightarrow \infty$ when $\|\boldsymbol{u}\| \rightarrow \infty \forall \boldsymbol{y} \in Y_{\text {ad }}$.

In what follows we deduce the optimality condition for a local minimum of the cost function $J(\boldsymbol{u}, \boldsymbol{y})$ given by (7.1). We notice that in the present case $J$ is convex with respect to the amplitudes $\boldsymbol{u}$ but not with respect to the actuators positions $\boldsymbol{y}$. Therefore, this optimality condition will be necessary but not sufficient.

The cost function can be written explicitly in terms of the control variables as follows:

$$
J(\boldsymbol{u}, \boldsymbol{y})=\frac{1}{2} \sum_{k=1}^{M}\left|p\left(w_{k}\right)\right|^{2}+\frac{\nu}{2}\|\boldsymbol{u}\|^{2}=\frac{1}{2} \sum_{k=1}^{M}\left|p_{0}\left(w_{k}\right)+\sum_{i=1}^{N} u_{i} G^{y_{i}}\left(w_{k}\right)\right|^{2}+\frac{\nu}{2} \sum_{i=1}^{N}\left|u_{i}\right|^{2} .
$$

This function is differentiable in $\mathbb{C}^{N} \times\left(\Omega \backslash\left\{w_{1}, \ldots, w_{M}\right\}\right)^{N}$. Hence, it is well known that if it attains a local minimum in the convex subset $U_{\text {ad }} \times Y_{\text {ad }}$ at $\left(\boldsymbol{u}^{\mathrm{op}}, \boldsymbol{y}^{\mathrm{op}}\right)$, then the following inequality holds:

$$
D J\left(\boldsymbol{u}^{\mathrm{op}}, \boldsymbol{y}^{\mathrm{op}}\right)\left(\boldsymbol{u}-\boldsymbol{u}^{\mathrm{op}}, \boldsymbol{y}-\boldsymbol{y}^{\mathrm{op}}\right) \geq 0 \quad \forall(\boldsymbol{u}, \boldsymbol{y}) \in U_{\mathrm{ad}} \times Y_{\mathrm{ad}} .
$$

Since $J$ depends on the complex variables $u_{1}, \ldots, u_{N}$, some care must be taken to compute its differential. Indeed, by using that

$$
D\left(\frac{1}{2}\|\boldsymbol{v}\|^{2}\right)(\delta \boldsymbol{v})=\operatorname{Re}\left(\overline{\boldsymbol{v}}^{\mathrm{t}} \delta \boldsymbol{v}\right)=\operatorname{Re}\left(\overline{\boldsymbol{v}}^{\mathrm{t}} \boldsymbol{v}\right) \quad \forall \boldsymbol{v}, \delta \boldsymbol{v} \in \mathbb{C}^{M} \text { or } \mathbb{C}^{N},
$$

straightforward computations lead to

$$
D_{\boldsymbol{u}} J(\boldsymbol{u}, \boldsymbol{y})(\delta \boldsymbol{u})=\operatorname{Re}\left\{\sum_{i=1}^{N} \sum_{k=1}^{M}\left[p\left(w_{k}\right) \frac{\partial \bar{p}\left(w_{k}\right)}{\partial u_{i}} \delta \bar{u}_{i}\right]+\nu \sum_{i=1}^{N} u_{i} \delta \bar{u}_{i}\right\},
$$

and

$$
D_{\boldsymbol{y}} J(\boldsymbol{u}, \boldsymbol{y})(\delta \boldsymbol{y})=\operatorname{Re}\left[\sum_{i=1}^{N} \sum_{k=1}^{M} p\left(w_{k}\right) \nabla_{y_{i}} \bar{p}\left(w_{k}\right) \cdot \delta y_{i}\right]
$$


$\forall(\boldsymbol{u}, \boldsymbol{y}) \in U_{\mathrm{ad}} \times Y_{\mathrm{ad}}, \forall \delta \boldsymbol{u} \in \mathbb{C}^{N}$, and $\forall \delta \boldsymbol{y} \in\left(\mathbb{R}^{n}\right)^{N}$. As a consequence of all this we obtain the optimality condition of the following theorem.

ThEOREM 7.1. If $\left(\boldsymbol{u}^{\mathrm{op}}, \boldsymbol{y}^{\mathrm{op}}\right) \in U_{\mathrm{ad}} \times Y_{\mathrm{ad}}$ is a solution of the control problem (7.1), then it satisfies

$$
\begin{aligned}
\operatorname{Re}\left\{\sum_{i=1}^{N} \sum_{k=1}^{M}\left[p\left(w_{k}\right) \frac{\partial \bar{p}\left(w_{k}\right)}{\partial u_{i}}\left(\bar{u}_{i}-\bar{u}_{i}^{\mathrm{op}}\right)+p\left(w_{k}\right) \nabla_{y_{i}} \bar{p}\left(w_{k}\right) \cdot\left(y_{i}-y_{i}^{\mathrm{op}}\right)\right]\right. \\
\left.+\nu \sum_{i=1}^{N} u_{i}^{\mathrm{op}}\left(\bar{u}_{i}-\bar{u}_{i}^{\mathrm{op}}\right)\right\} \\
\geq 0 \quad \forall(\boldsymbol{u}, \boldsymbol{y}) \in U_{\mathrm{ad}} \times Y_{\mathrm{ad}} .
\end{aligned}
$$

Standard duality arguments can be used to compute the gradient of the cost function. To this aim, given $(\boldsymbol{u}, \boldsymbol{y}) \in U_{\mathrm{ad}} \times Y_{\mathrm{ad}}$, we recall the state equation:

$$
\left\{\begin{aligned}
-\Delta p-\left(\frac{\omega}{c}\right)^{2} p & =\sum_{i=1}^{N} u_{i} \delta_{y_{i}} & & \text { in } \Omega \\
\frac{\partial p}{\partial \boldsymbol{n}} & =\frac{i \omega \rho}{Z(\omega)} p & & \text { on } \Gamma_{\mathrm{Z}} \\
\frac{\partial p}{\partial \boldsymbol{n}} & =g & & \text { on } \Gamma_{\mathrm{N}}
\end{aligned}\right.
$$

and introduce the adjoint state equation:

$$
\left\{\begin{aligned}
-\Delta r-\left(\frac{\omega}{c}\right)^{2} r & =\sum_{k=1}^{M} p\left(w_{k}\right) \delta_{w_{k}} & & \text { in } \Omega, \\
\frac{\partial r}{\partial \boldsymbol{n}} & =-\frac{i \omega \rho}{\bar{Z}(\omega)} r & & \text { on } \Gamma_{\mathrm{z}}, \\
\frac{\partial r}{\partial \boldsymbol{n}} & =0 & & \text { on } \Gamma_{\mathrm{N}},
\end{aligned}\right.
$$

with $p$ being the solution of the state equation (7.4). Then we have the following result.

Theorem 7.2. Let $(\boldsymbol{u}, \boldsymbol{y}) \in U_{\mathrm{ad}} \times Y_{\mathrm{ad}}$, and $(\delta \boldsymbol{u}, \delta \boldsymbol{y}) \in \mathbb{C}^{N} \times\left(\mathbb{R}^{n}\right)^{N}$. Let $p$ be the solution of of the state equation (7.4) and $r$ be the solution of the adjoint state equation (7.5). Then,

$$
D_{\boldsymbol{u}} J(\boldsymbol{u}, \boldsymbol{y})(\delta \boldsymbol{u})=\operatorname{Re}\left\{\sum_{i=1}^{N}\left[r\left(y_{i}\right)+\nu u_{i}\right] \delta \bar{u}_{i}\right\},
$$

and

$$
D_{\boldsymbol{y}} J(\boldsymbol{u}, \boldsymbol{y})(\delta \boldsymbol{u})=\operatorname{Re}\left[\sum_{i=1}^{N} \bar{u}_{i} \nabla r\left(y_{i}\right) \cdot \delta y_{i}\right] .
$$

Proof. Let $G^{w_{k}}$ be the solution of problem (3.6) with $y=w_{k}, k=1, \ldots, M$. Then

$$
r=\sum_{k=1}^{M} p\left(w_{k}\right) \bar{G}^{w_{k}}
$$


On the other hand, from (5.1), for $w_{k} \neq y_{i}, i=1, \ldots, N, k=1, \ldots, M$,

$$
\frac{\partial \bar{p}\left(w_{k}\right)}{\partial u_{i}}=\frac{\partial}{\partial u_{i}}\left[\bar{p}_{0}\left(w_{k}\right)+\sum_{i=1}^{N} u_{i} \bar{G}^{y_{i}}\left(w_{k}\right)\right]=\bar{G}^{y_{i}}\left(w_{k}\right)=\bar{G}^{w_{k}}\left(y_{i}\right)
$$

and

$$
\nabla_{y_{i}} \bar{p}\left(w_{k}\right)=\bar{u}_{i} \nabla_{y_{i}} \bar{G}^{y_{i}}\left(w_{k}\right)=\bar{u}_{i} \nabla \bar{G}^{w_{k}}\left(y_{i}\right)
$$

where we have used the symmetry of $G^{y}(w)$ with respect to $y$ and $w$ in $\Omega$.

Consequently, from (7.2) and (7.3) we have

$$
\begin{aligned}
D_{\boldsymbol{u}} J(\boldsymbol{u}, \boldsymbol{y})(\delta \boldsymbol{u}) & =\operatorname{Re}\left\{\sum_{i=1}^{N} \sum_{k=1}^{M}\left[p\left(w_{k}\right) \bar{G}^{w_{k}}\left(y_{i}\right) \delta \bar{u}_{i}\right]+\nu \sum_{i=1}^{N} u_{i} \delta \bar{u}_{i}\right\} \\
& =\operatorname{Re}\left\{\sum_{i=1}^{N}\left[r\left(y_{i}\right)+\nu u_{i}\right] \delta \bar{u}_{i}\right\},
\end{aligned}
$$

and

$$
D_{\boldsymbol{y}} J(\boldsymbol{u}, \boldsymbol{y})(\delta \boldsymbol{y})=\operatorname{Re}\left[\sum_{i=1}^{N} \sum_{k=1}^{M} p\left(w_{k}\right) \bar{u}_{i} \nabla \bar{G}^{w_{k}}\left(y_{i}\right) \cdot \delta y_{i}\right]=\operatorname{Re}\left[\sum_{i=1}^{N} \bar{u}_{i} \nabla r\left(y_{i}\right) \cdot \delta y_{i}\right] .
$$

Thus we conclude the proof. $\mathrm{C}$

As a consequence of this theorem, we can write the optimality condition of problem (7.1) in terms of the the solution $p$ of the state equation and the solution $r$ of the adjoint state equation. Thus we obtain the following Euler's inequality:

$$
\begin{aligned}
& \operatorname{Re}\left(\sum_{i=1}^{N}\left\{\left[r\left(y_{i}^{\mathrm{op}}\right)+\nu u_{i}^{\mathrm{op}}\right]\left(\bar{u}_{i}-\bar{u}_{i}^{\mathrm{op}}\right)+\bar{u}_{i}^{\mathrm{op}} \nabla r\left(y_{i}^{\mathrm{op}}\right) \cdot\left(y_{i}-y_{i}^{\mathrm{op}}\right)\right\}\right) \geq 0 \\
& \forall(\boldsymbol{u}, \boldsymbol{y}) \in U_{\mathrm{ad}} \times Y_{\mathrm{ad}} .
\end{aligned}
$$

7.2. Numerical experiments. In this section we present two numerical tests. The goal of the first one is to show that many local minima can actually arise. It is a one-dimensional problem and, then, the Helmholtz equation becomes

$$
\left\{\begin{aligned}
-\frac{d^{2} p}{d x^{2}}-\left(\frac{\omega}{c}\right)^{2} p & =f & & x \in(a, b), \\
\frac{d p}{d x} & =\frac{i \omega \rho}{Z(\omega)} p & & \text { at } x=a, \\
\frac{d p}{d x} & =g & & \text { at } x=b .
\end{aligned}\right.
$$

This $1 \mathrm{D}$ equation can be easily solved when the secondary source is a linear combination of Dirac delta measures,

$$
f=\sum_{i=1}^{N} u_{i} \delta_{y_{i}}, \quad u_{i} \in \mathbb{C}, y_{i} \in(a, b), i=1, \ldots, N .
$$

Thus, we can determine the corresponding optimal amplitudes and then the optimal value of the cost function.

First, we consider the following data: 
- the domain is the segment $[0,1] \mathrm{m}$;

- the physical parameters are $\rho=1 \mathrm{~kg} \mathrm{~m}^{-3}, c=340 \mathrm{~m} \mathrm{~s}^{-1}$, and $\omega=18700 \mathrm{~s}^{-1}$;

- the amplitude of the primary source is $g=1 \mathrm{~kg} \mathrm{~m}^{-2} \mathrm{~s}^{-2}$ at $x=1 \mathrm{~m}$;

- the wall impedance at $x=0$ is $Z=34 \times 10^{7}+34 \times 10^{3} i \mathrm{~kg} \mathrm{~m}^{-2} \mathrm{~s}^{-1}$;

- there is one actuator located at any point in the segment $Y_{\text {ad }}=[0.4,0.6] \mathrm{m}$;

- there are 4 sensors at the points $w_{1}=0.15 \mathrm{~m}, w_{2}=0.25 \mathrm{~m}, w_{3}=0.65 \mathrm{~m}$, and $w_{4}=0.85 \mathrm{~m} ;$

- the admissible set of amplitudes is $U_{\text {ad }}=\mathbb{C}$ and the weighting factor is $\nu=0$.

For each position of the actuator in $Y_{\mathrm{ad}}$, we compute the optimal amplitude and represent the corresponding value of the cost function. Figure 7.1 shows this function. We observe several local minima. Furthermore, in this case, the values of $J$ at all these minima are the same and correspond to maximum values of the optimal amplitude.

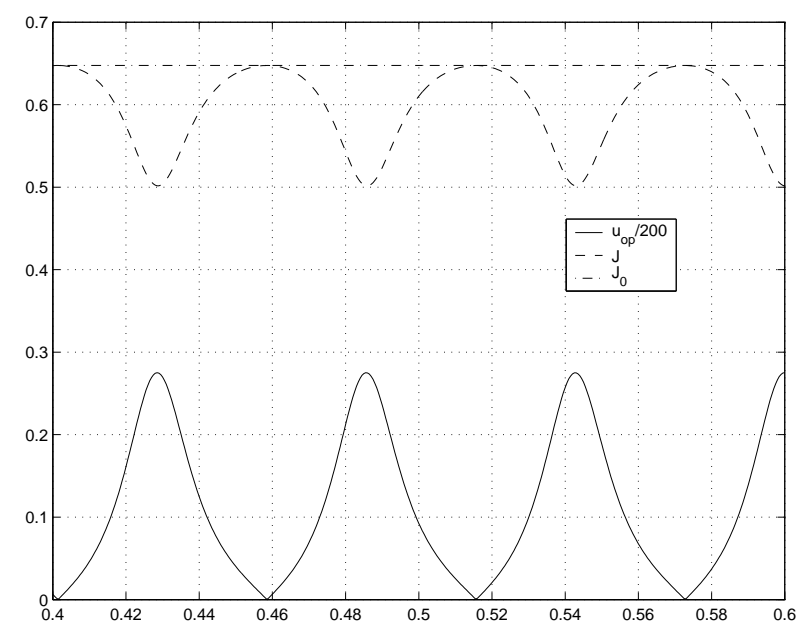

FIG. 7.1. $J$ and $u_{\mathrm{op}}$ as functions of the loud-speaker position $\left(\omega=18700 \mathrm{~s}^{-1}\right)$.

Second, we analyze the system as the angular frequency decreases. Figures 7.2 and 7.3 show similar graphs for $\omega=7820 \mathrm{~s}^{-1}$ and $\omega=1700 \mathrm{~s}^{-1}$, respectively.

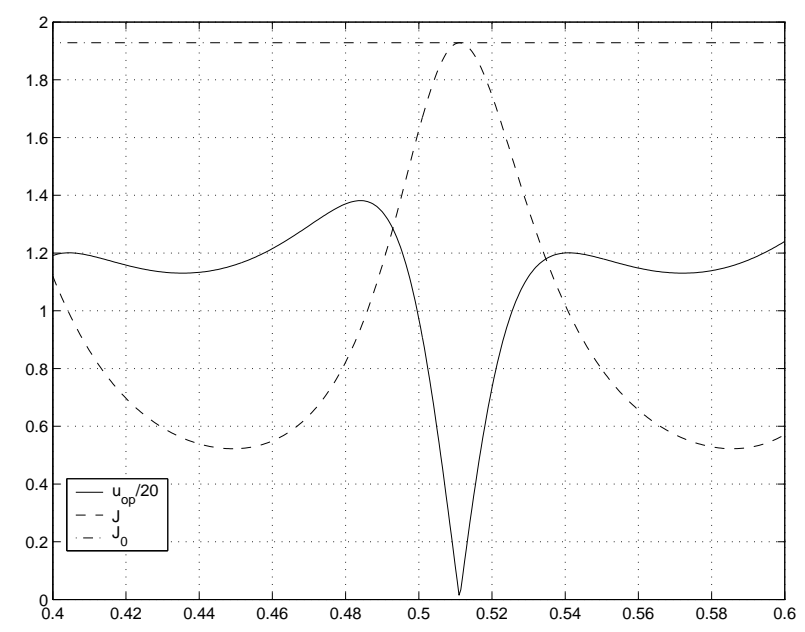

FiG. 7.2. $J$ and $u_{\mathrm{op}}$ as functions of the loud-speaker position $\left(\omega=7820 \mathrm{~s}^{-1}\right)$. 


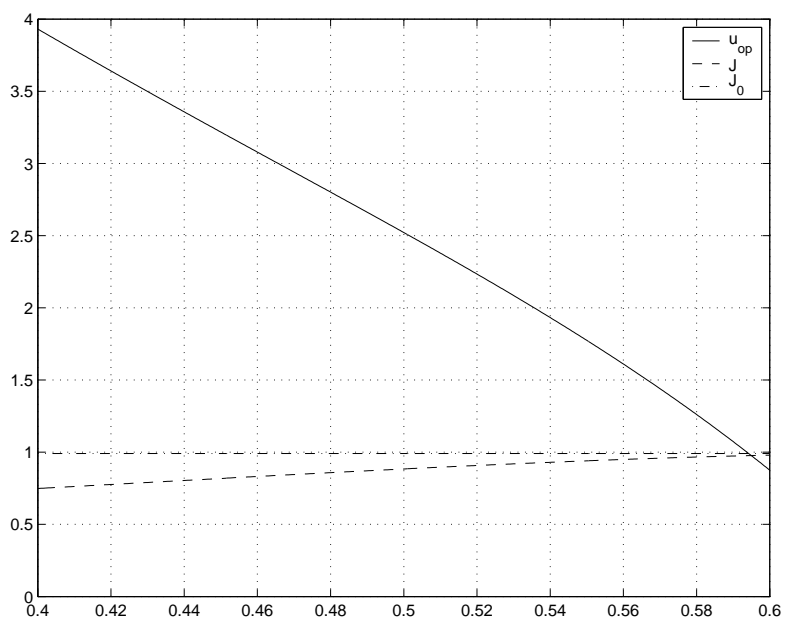

FIG. 7.3. $J$ and $u_{\mathrm{op}}$ as functions of the loud-speaker position $\left(\omega=1700 \mathrm{~s}^{-1}\right)$.

We observe in Figure 7.2 that the local minima of the cost functional do not necessarily coincide with local maxima of the optimal amplitude.

We also notice that the number of local minima diminishes with the angular frequency. For instance, in the case of Figure $7.3, J$ has only one minimum in the interval $Y_{\text {ad }}=[0.4,0.6] \mathrm{m}$.

Figure 7.4 shows the corresponding graph for the same frequency $\omega=1700 \mathrm{~s}^{-1}$, when the admissible set of locations for the actuator is the whole domain of the problem: $Y_{\mathrm{ad}}=[0,1] \mathrm{m}$. In this case it can be seen that, as expected, complete attenuation is attained as the actuator gets close to the primary source at $x=1$.

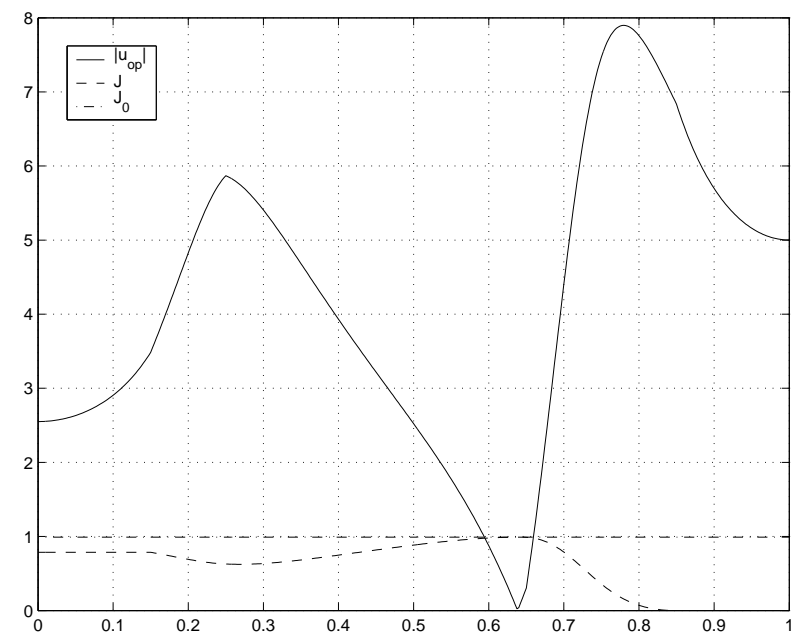

FIG. 7.4. $J$ and $u_{\mathrm{op}}$ as functions of the loud-speaker position $\left(\omega=1700 \mathrm{~s}^{-1}, Y_{\mathrm{ad}}=[0,1] \mathrm{m}\right)$.

The second test corresponds to a three-dimensional enclosure. We use the simulated annealing algorithm (see for instance [1]) to determine the optimal location of loud-speakers among a given finite number of feasible ones.

The data of the test are the following:

- domain $\Omega=[0,1] \mathrm{m} \times[0,1] \mathrm{m} \times[0,1] \mathrm{m}$; 
- physical parameters $\rho=1 \mathrm{~kg} \mathrm{~m}^{-3}, c=340 \mathrm{~m} \mathrm{~s}^{-1}$, and $\omega=1360 \mathrm{~s}^{-1}$;

- wall impedance at $z=1 \mathrm{~m}, Z=(102+340 i) \times 10^{3} \mathrm{~kg} \mathrm{~m}^{-2} \mathrm{~s}^{-1}$;

- primary source at wall $z=0$ with amplitude $g(x, y, 0)=\mathrm{e}^{i y} \mathrm{~kg} \mathrm{~m}^{-2} \mathrm{~s}^{-2}$;

- the rest of the walls are perfectly rigid;

- we have to locate 8 actuators and consider 16 possible locations which are shown in Table 7.1;

- there are 10 sensors and their positions are shown in Table 7.2;

- admissible set of amplitudes $U_{\mathrm{ad}}=\mathbb{C}^{8}$ and weighting factor $\nu=0$.

We have used a mesh like that of Figure 6.1 for $h=1 / 24 \mathrm{~m}$.

TABLE 7.1

Possible actuator locations.

\begin{tabular}{|c|c|c|c|}
\hline Coordinates $(\mathrm{m})$ & Coordinates $(\mathrm{m})$ & Coordinates $(\mathrm{m})$ & Coordinates $(\mathrm{m})$ \\
\hline$(0.1,0.1,0.1)$ & $(0.5,0.4,0.1)$ & $(0.5,0.1,0.3)$ & $(0.8,0.8,0.3)$ \\
\hline$(0.9,0.9,0.1)$ & $(0.5,0.6,0.1)$ & $(0.5,0.9,0.3)$ & $(0.6,0.4,0.4)$ \\
\hline$(0.1,0.9,0.1)$ & $(0.1,0.5,0.3)$ & $(0.5,0.5,0.3)$ & $(0.4,0.6,0.4)$ \\
\hline$(0.9,0.1,0.1)$ & $(0.9,0.5,0.3)$ & $(0.2,0.2,0.3)$ & $(0.6,0.6,0.4)$ \\
\hline
\end{tabular}

TABLE 7.2

Sensor positions.

\begin{tabular}{|c|c|}
\hline Coordinates $(\mathrm{m})$ & Coordinates $(\mathrm{m})$ \\
\hline$(0.2,0.2,0.6)$ & $(0.5,0.1,0.8)$ \\
\hline$(0.2,0.8,0.6)$ & $(0.1,0.5,0.8)$ \\
\hline$(0.8,0.2,0.6)$ & $(0.5,0.9,0.8)$ \\
\hline$(0.8,0.8,0.6)$ & $(0.7,0.5,0.6)$ \\
\hline$(0.9,0.5,0.8)$ & $(0.3,0.5,0.8)$ \\
\hline
\end{tabular}

The attenuation is computed again by

$$
\text { Attenuation }(\mathrm{dB})=-10 \log _{10}\left(\frac{J\left(\boldsymbol{u}^{\mathrm{op}}, \boldsymbol{y}^{\mathrm{op}}\right)}{J(\mathbf{0}, \boldsymbol{y})}\right) \text {. }
$$

Notice that $J(\mathbf{0}, \boldsymbol{y})$ is the value of the cost function with no control, and hence it does not depend on $\boldsymbol{y}$. In Table 7.3 we show the attenuation obtained for different executions of the simulated annealing algorithm. We also include the value obtained with the exhaustive search, i.e., by computing the cost function for all of the possible $\left(\begin{array}{c}16 \\ 8\end{array}\right)=12870$ configurations.

TABLE 7.3

Simulated annealing: number of iterations and optimal attenuation.

\begin{tabular}{|c|c|}
\hline No. of Iterations & Attenuation $(\mathrm{dB})$ \\
\hline 569 & 73.7 \\
\hline 599 & 73.7 \\
\hline 710 & 73.7 \\
\hline 785 & 73.7 \\
\hline 800 & 68.6 \\
\hline 1201 & 73.7 \\
\hline 1498 & 73.7 \\
\hline \hline 12870 (exhaustive) & 73.7 \\
\hline
\end{tabular}

REMARK 3. Some experiments show that the use of a basis consisting of rigid cavity vibration modes can be more efficient in terms of computer effort (CPU time 
and memory) than the purely finite element technique introduced in this paper. This is due to the fact that the number of vibration modes needed to obtain accurate results for low frequencies (which is the typical case in active control of sound) is not large. Then the time to calculate these first modes by solving the corresponding eigenvalue problem by finite element methods, together with that to solve the Helmholtz problems in this small vibration modes basis, can be significantly less than the time needed to solve the same number of Helmholtz problem in the large finite element basis. This approach will be reported elsewhere.

Acknowledgments. Authors wish to thank Dr. Pedro Cobo from IAC/CSIC, Madrid (Spain) for very useful discussions.

This work was partially done while one of the authors (R.R.) participated in the programme Computational Challenges in PDEs at the Newton Institute of the University of Cambridge, on June 2003. He is grateful for the support and stimulating atmosphere at the Institute.

Last but not least, the authors wish to thank Ricardo Durán and Ricardo Nochetto, for very helpful discussions.

\section{REFERENCES}

[1] K. H. Baek And S. J. Elliot, Natural algorithms for choosing source locations in active control systems, J. Sound and Vibr., 186 (1995), pp. 245-267.

[2] S. Brenner, L. R. Scott, Mixed and Hybrid Finite Element Methods, Springer, New York, Berlin, Heidelberg, 1996

[3] L. L. Beranek and I. L. Ver, Noise and Vibration Control Engineering. Principles and Applications, John Wiley and Sons, New York, 1992.

[4] E. CASAS, $L^{2}$ estimates for the finite element method for the Dirichlet problem with singular data, Numer. Math., 47 (1985), pp. 627-632.

[5] M. Dauge, Elliptic Boundary Value Problems on Corner Domains: Smoothness and Asymptotics of Solutions, Lecture Notes in Mathematics, 1341, Springer-Verlag, 1988.

[6] R. Dautray and J. L. Lions, Mathematical Analysis and Numerical Methods for Science and Technology, Springer-Verlag, Berlin, 1984-1985.

[7] L. C. Evans, Partial Differential Equations, American Mathematical Society, Providence, RI, 1998.

[8] P. Gamallo, Contribución al estudio matemático de problemas de simulación elastoacústica $y$ control activo del ruido. PhD. Thesis. Universidade de Santiago de Compostela, Spain, 2002.

[9] P. Grisvard, Elliptic Problems for Non-smooth Domains, Pitman, 1985.

[10] W. Hackbusch, Elliptic Partial Differential Equations, Theory and Numerical Treatment, Springer-Verlag, Berlin, Heidelberg, New York, 1992.

[11] P. A. Nelson And S. J. Elliot, Active Control of Sound, Academic Press, London, 1999.

[12] A. Schatz, An observation concerning Ritz-Galerkin methods with indefinite bilinear forms, Math. Comp., 28 (1974), pp. 959-962.

[13] A. Schatz And L. WAhlbin, Interior maximum norm estimates for finite element methods, Math. Comp., 31 (1977), pp. 414-442.

[14] R. Sсотт, Finite element convergence for singular data, Numer. Math., 21 (1973), pp. 317-327. 